\title{
Clinical Spectroscopy: Lost in Translation?
}

James M. Cameron ${ }^{1}$, Christopher Rinaldi ${ }^{2}$, Samantha H. Rutherford ${ }^{2}$, Alexandra Sala ${ }^{2}$, Ashton G. Theakstone ${ }^{2}$, Matthew J. Baker ${ }^{1}$

${ }^{1}$ ClinSpec Diagnostics Ltd, Royal College Building, 204 George Street, Glasgow, G1 1XW, UK

${ }^{2}$ WestCHEM, Department of Pure and Applied Chemistry, Technology and Innovation Centre, 99 George Street, Glasgow, G1 1RD, UK

Corresponding author: matthew.baker@clinspecdx.com; Twitter; @chemistrybaker

\begin{abstract}
$\underline{\text { Abstract }}$
This focal point article discusses the developments of biomedical Raman and infrared spectroscopy, and the recent strive towards being a recognised clinical tool for various applications. The promise of vibrational spectroscopy in the field of biomedical science, alongside the development of computational methods for spectral analysis, has driven a plethora of proof-of-concept studies which convey the potential of various spectroscopic approaches. Here we report a brief review of the literature published over the past few decades, with a focus on the current technical, clinical and economic barriers to translation, namely the limitations of many of these early studies, the lack of understanding of clinical pathways, health technology assessments, regulatory approval, clinical feasibility and funding applications. The field of biomedical vibrational spectroscopy must acknowledge and overcome these hurdles in order to achieve clinical efficacy. Current prospects have been overviewed with comment on the advised future direction of spectroscopic technologies, with the aspiration that many of these innovative approaches can ultimately reach the frontier of medical diagnostics and other clinical applications.
\end{abstract}

This is a peer-reviewed, accepted author manuscript of the following article: Cameron, J. M., Rinaldi, C., Rutherford, S. H., Sala, A., G. Theakstone, A., \& Baker, M. J. (2021). Clinical spectroscopy: lost in translation? Applied Spectroscopy. https://doi.org/10.1177/00037028211021846 


\section{Introduction}

The use of vibrational spectroscopy, namely Raman and infrared (IR), has been well proven over the past $50+$ years, showing its abilities to accurately characterise clinical and biological specimens and provide useful information on the disease status of the specimens. Accuracy and potential of the technique have been the foundation of a vast amount of global research targeted towards solving issues within disease identification, analysis, prognosis and therapy for human good which has enabled strides forward in measurement techniques, technologies and our fundamental understanding of the interaction of electromagnetic radiation with matter.

In 1997, Jackson et al. published a review entitled "Infrared Spectroscopy: a new frontier in medicine" with a call to action: "As we enter the second half of the nineties, one of the major challenges for biological infrared spectroscopists is to transfer the knowledge we have gained from studies on isolate molecules to the complex world of medicine. That it is possible to meet this challenge is suggested by comparison with the development of other biophysical techniques, such as magnetic resonance spectroscopy and imaging, which have already found their place in medical research and practice" ${ }^{1}$.

Magnetic resonance (MR) spectroscopy and magnetic resonance imaging (MRI) are valuable comparators for clinical vibrational spectroscopy. MRI is a non-invasive method of mapping the internal structure and certain functions within the body ${ }^{2}$. The technique uses radio frequency radiation in the presence of carefully controlled magnetic fields in order to provide this information. MRI has evolved from being a technique with great potential, to one that has become the primary diagnostic investigation for many medical complications ${ }^{3}$. In fact, in 1971 Raymond Damadian suggested that MR relaxation times could be used to detect cancerous tissue ${ }^{4}$. Lauterbur published his work in Nature in 1973, on how to use NMR to obtain images in macroscopic dimensions ${ }^{5}$. The first human images were able to be taken in $1977^{6}$, and since then MRI has become a primary medical tool. Off the back of their ground-breaking research in the 1970s, the 2003 Nobel Prize in Physiology or Medicine was jointly awarded to Lauterbur and Mansfield for their discoveries concerning magnetic resonance imaging ${ }^{7}$ which was by then fully utilised in the clinic.

A web of knowledge search for publications including the terms Fourier transform infrared (FTIR) or Raman when combined with 'clinical', 'biology' or 'medicine', with a date range between 1960 and 2020, is illustrated in Figure 1. The exponential increase in publications over 
time clearly demonstrates the evolution of a rapidly expanding research field, acknowledging the first related publication in 1978 .

$<$ Insert Figure 1 here $>$

In light of this excellent body of research combined with the concerted global effort to translate spectroscopy to the clinic, and the comparison with MRI in the 1990s, we have to consider and evaluate the reasons why vibrational spectroscopy is yet to become an acknowledged clinical tool. Various spectroscopic societies have been formed in the past few decades, paving the way for many annual and biannual conferences for experts in the field to monitor and discuss the necessities to achieve clinical translation.

A Faraday Discussion is a prestigious conference series organised by the Royal Society of Chemistry, UK. These meetings are targeted at fast-growing areas of the physical sciences, to act as a record of international opinion in the area and uniquely publish the debates alongside the discussion papers. A Faraday Discussion for a subject area is awarded after a written proposal and aims to drive that area forward through scientific discourse. Our research area has been the subject of two Faraday Discussions. In 2004, Jackson gave the introductory lecture at a Faraday Discussion entitled "From biomolecules to biodiagnostics: Spectroscopy does it all" and highlighted in his paper reasons for the apparent slow penetration of optical diagnostics into clinical practice ${ }^{8}$. Specifically, the causes for lack of penetration were highlighted as:

1) Biological spectroscopy is not progressing quickly, rather the published work is more of the same and a lack of imagination from researchers;

2) Quality of studies compromised by a "publish or perish" culture;

3) Quality of studies compromised by lack of statistical power;

4) Lack of health economic evidence of potential impact for financial viability;

5) No published long-term, multi-site, multi-instrument studies that validate optical technology for clinical use. 
In the 2016 Faraday Discussion, Petrich gave the closing lecture entitled "Translating vibrational spectroscopy into clinical applications - vision or reality?", highlighting that there has been progress in clinical application and that biomedical vibrational spectroscopy has reached a pivotal point at which questions like diagnostic value, therapeutic consequence and financial viability are becoming paramount ${ }^{9}$. Petrich concluded by focusing upon a series of questions that we need to answer from a healthcare standpoint:

1) What is the unmet need?

2) What precisely is the diagnostic value?

3) What is the therapeutic consequence?

4) Will this application be financially viable?

In a 2015 paper which was linked to SPEC2014, Byrne et al. addressed the question "Quo Vadis?" (where are you going?) for the next generation of spectropathology ${ }^{10}$. This article was based on discussion around the specific topics of: (i) What are the most achievable, strategic target applications?; (ii) What are the technical challenges, and how can they be addressed?; and (iii) What are the challenges to implementation (legislative, clinical trials etc.), and how can they be addressed? The SPEC conference series, which began in 2000, is now considered the flagship of the International Society for Clinical Spectroscopy (CLIRSPEC). The authors also highlighted the need to:

1) Enable a more significant engagement between the broad range of stakeholders (academia, industry and clinical);

2) Establishing and promoting standard operating procedures (SOPs), for measurement and data handling protocols;

3) More targeted engagement with the medical community undertaken to establish strategic target applications and performance levels for minimal viable solutions;

4) Funding needed to conduct large-scale, multi-lab and -instrument intercomparisons and ultimately clinical trials.

The establishment of various peer-reviewed journals in the field - such as the Journal of Biophotonics, Translational Biophotonics and Clinical Spectroscopy - has provided a valuable platform for spectroscopic researchers to showcase their potential for clinical translation. Furthermore, it is worth noting the formation of multinational networks, namely CLIRSPEC and Raman4Clinics. CLIRSPEC is a non-profit organisation, founded in 2015, that aims to act 
as a platform for these individuals, teams and organisations wishing to promote the translation for spectroscopy into the clinical environment ${ }^{11}$. The society covers a wide range of spectroscopy techniques with the overarching aim of the collaboration to improve patient diagnosis and prognosis ${ }^{11}$. Raman4Clinics, again founded in 2015, pools European expertise to progress in the field of novel, label-free and rapid technologies based on a wide variety of Raman spectroscopy for the clinical diagnostics of body fluids, bacteria, cells and tissues ${ }^{12}$. Raman4Clinics is a European platform that combines six working groups and numerous partners aiming for clinical diagnostics of biological fluids, bacteria, cells and tissues utilising a wide range of Raman spectroscopy techniques ${ }^{12}$. Each working group is designed to target separate aims including therapeutic drug monitoring, identification of microbial pathogens, cancer cell monitoring, histopathology, in vivo assessment and outreach to improve public and industry awareness. Raman4Clinics combines the use of non-destructive and tuneable Raman spectroscopy with microscopic techniques for mapping, as well as fibre optic probes for minimal invasive endoscopies, as described in their 'European Cooperation in the field of Scientific and Technical Research' (COST) action plan, published in 2014 and completed in $2018^{13}$.

The process of technology transfer from the laboratory bench is of course not simple, rather a multifaceted challenge that requires expertise from a wide range of different disciplines. An article published by Morris et al. highlights that several studies have converge around an average time lag of 17 years from research evidence to move through to clinical practice ${ }^{14}$. However, the authors warned that these studies use different metrics and a greater understanding of the reasons behind the time-lag would be beneficial for all, including investment decisions. A conceptual matrix for measuring and understanding the time lags suggested by Hanney et al. is shown in Figure 2 below ${ }^{15}$.

$<$ Insert Figure 2 here $>$

The current state of the translation field is described by Figure 2, where most of the research performed within clinical spectroscopy is still on the discovery research track concerned with "most relevant discovery research". The field has managed to generate some level of investigation within the 'human research and review' tracks, yet there is very little research 
within our discipline that could be considered in the 'clinical and health service public policy development' tracks. We are now at the beginning of the third decade of the $21^{\text {st }}$ Century and vibrational spectroscopy is still not currently used as a standard clinical tool. This focal point review will discuss and assess recent progress in the area, in light of the highlighted needs from the position papers described above. 


\section{$\underline{\text { Proof-of-concept Studies for Biomedical Applications }}$}

\section{Tissues \& Cells}

Over the years, applications of vibrational spectroscopy techniques have been tested and developed as potential tools for disease diagnostics due to their high specificity and ability to cover a broad range of molecular species ${ }^{16-18}$. Many diseases lead to changes at a molecular level ${ }^{19-21}$, whether that be a change in the chemical compositional or molecular structures within biofluids, tissues or cells, making spectroscopic techniques ideal for the detection of many different disease states ${ }^{22-25}$.

In the field of oncology, tissue specimens are often judged by a pathologist to determine the stage and grade of a tumour. Typically, this is an extensive and subjective process involving assessment of the morphological features, requiring histological preparations, and does not utilise biochemical information at a cellular level ${ }^{26-28}$. However, vibrational spectroscopy techniques provide a biochemical fingerprint of the sample and several proof-of-concept studies have shown the ability of these techniques to assess and diagnose tissue samples faster than conventional methods ${ }^{29-34}$.

Gajjar et al. utilised glioma brain tissue enabled discrimination between glioma subtypes using both attenuated total reflection Fourier transform infrared (ATR-FTIR) and Raman spectroscopies ${ }^{35}$. Spectral biomarkers were identified using both modalities, of most prominence was a lipid-to-protein ratio, with a notable decreasing trend recorded with the progression of gliomas from healthy tissue to most severe. Several other ratios exploiting different chemical biomarkers were also identified in each of the modalities to help determine between glioma subtypes. Results obtained in this study were comparable to those from histopathology staining techniques, yet on a much quicker timescale.

A number of early studies investigated vibrational spectroscopy as a tool for aiding the detection of cervical cancer. This area has since expanded dramatically with more recent studies probing the spectral response of cervical cancer cell lines to human papillomavirus ${ }^{36-}$ ${ }^{38}$. Furthermore, cells exfoliated from oral tissues have also been shown to be effective in the diagnosis of oropharyngeal and laryngeal cancers ${ }^{39}$. In a bid to develop a diagnostic tool for lung cancer, FTIR analysis of sputum cell pellets created from 50 patients' sputum samples was published by Lewis et al. ${ }^{40}$. The FTIR measurements of the cell pellets revealed significant 
differences between the IR spectrum of samples from patients suffering with lung cancer and healthy controls. In particular, tumour cells showed elevated signals of proteins, glycogen and nucleic acids which led to their identification from controls by multivariate analysis methods, with accuracies $>92 \%$. A number of aspects of spectrocytology make it well suited to the clinic; samples are generally easy to obtain and the opportunity to adjunct onto existing screening programmes make it an attractive target for translation. However, there remains no clear progression towards a clinical setting.

For the analysis of tissue and cell samples, IR spectroscopies can be combined with microscopy techniques offering label-free and rapid mapping of large areas of biopsies, enabling high sensitivity and admirable spatial resolution $(\sim 1-10 \mu \mathrm{m})$. IR spectral histopathology collects IR spectra across thousands of pixels allowing a 'hyperspectral datacube' to be built for each tissue specimen. One study employing the use of artificial neural network (ANN) machine learning algorithms, highlighted the power of IR microscopy for the classification of lung cancer ${ }^{41}$. An accuracy of $96.8 \%$ was achieved in identifying cancerous vs non-cancerous tissues. Additionally, small cell lung carcinomas, squamous cell carcinomas and adenocarcinomas were also classified with an average accuracy of $95 \%$. In an attempt to better understand lung cancer, an FTIR microspectroscopy study of lung cancer cells was carried out to assess their response to chemotherapy cancer treatment ${ }^{42}$. Addition of the chemotherapy drug showed clear indication of a halt in the cancer cell proliferation, in the form of a ratio associated with carbohydrates moieties and glycogen. The increase in this ratio was shown to be linearly correlated with the cells' survival after treatment, presenting a means of monitoring cancer cells in vitro.

Like many cancers, treatment outcomes for colon cancer are critically dependant on the accuracy of the final histopathology diagnosis. An IR microscopy study of 77 patients' colon samples showed differentiation of normal and malignant features with very promising results 27. This study highlighted the potential of a complementary objective biochemical diagnostic approach allowing a reduction in human involvement, thus speeding up the diagnosis process. Furthermore, an FTIR microscopy study of colon tissues combined with random forest (RF) classification has identified particular IR regions of interest, pertaining to $\mathrm{CH}$ stretching mode of lipids and the phosphate asymmetric stretch of nucleic acids, for the differentiation between cancerous and non-cancerous colon samples with accuracies greater than $90 \%{ }^{43}$. 
Combining an FTIR microspectroscopic system with a synchrotron light source for a broadband high brightness allows high spatial resolution images to be obtained, suitable for a single cell measurement. The application of this technique has shown significant potential to help understand disease formation and progression ${ }^{44-46}$. This synchrotron technique has been employed using the ATR configuration and was found to be able to identify a single malaria infected red blood cell (RBC) ${ }^{45}$. Chemical characterisation of the infected cells showed a decrease in haemoglobin levels in infected cells as well as the presence of haemoglobin byproducts. ATR-FTIR spectroscopy studies led by Wood et al. have also shown successful detection of RBCs infected with malaria ${ }^{45,47-50}$. One study in particular quantifies the parasite even in its early stages with an absolute detection limit of 1 infected RBC per microlitre of blood $^{50}$.

Delineating tumour tissue from healthy tissue during surgery is challenging, thus accurate tumour margins can be difficult to obtain for some cancers. Often adjuvant therapies or further imaging and surgeries are needed before the entire tumour is removed. Due to its compatibility with fibres and low water signal, Raman spectroscopy based intra-operative imaging techniques have been developed, allowing rapid assessment of tumour margins and help assist surgeons in real time ${ }^{51,52}$. This provides in situ analysis of tissues allowing excision of only the cancerous tumour, leaving as much healthy tissue as possible. Maximal safe resection decreases the likelihood of further surgeries to completely excise the tumour and generally improves prognosis for the patients. The ability to differentiate between cancerous and noncancerous bladder tissue has been illustrated by using a compact fibre-based Raman probe imaging system ${ }^{53}$. The study led by Cordero et al. facilitates real time evaluation of bladder tissue, enabling rapid analysis and diagnosis. Prediction models using linear discriminant analysis coupled with partial least squares (PLS-DA) resulted in a sensitivity and specificity of $92 \%$ and $93 \%$, respectively. Furthermore, through biochemical characterisations in the form of peak assignments can be made to also determine the bladder tumour grade, allowing a more accurate treatment plan and prognosis to be made. Kong et al. examined the potential of a Raman-based intra-operative evaluation of tumour excision, during breast conserving surgery (Figure 3$)^{54}$. The tumour regions were discriminated from the healthy tissue structures based on increased concentration of nucleic acids and reduced concentration of collagen and fat. The authors concluded that the technique has the potential to detect mammary carcinoma in tissue 
and assess resection margins, as well as rapid intra-operative assessment of sentinel axillary lymph node biopsies during breast cancer surgery.

$<$ Insert Figure 3 here $>$

Other techniques have been developed to utilise Raman spectroscopy in a bid to obtain accurate diagnoses from needle biopsies. It is widely known that multiple needle biopsies are necessary to obtain quality tissue for an accurate conclusion of disease state, and patients are at increasing risk of infections if they have to undergo multiple biopsies. Targeted in vivo Raman methods have been developed to guide surgeries, in order to acquire biopsies at the correct tissue location and/or to maximise cancer resection, providing cancer characterisation in situ ${ }^{55-58}$. An in vivo study involving 19 patients with gliomas between grades II-IV has highlighted the potential of an intra-operative Raman probe during brain surgery to successfully detect cancer tissue from non-cancerous tissue ${ }^{55}$. Following spectroscopic interrogation, a tissue biopsy was collected from the same area and evaluated by a neuropathologist. Based on a threshold of $60 \%$ of cells showing signs of cancer, high values of accuracy were produced using a support vector machine (SVM) approach, with 141 spectral features for optimum discrimination. The spectroscopic data can be collected instantaneously and offers significant advantage over pathology reports. Raman probes have also been utilised to assess and compare in vivo Raman spectra of skin legions and normal healthy skin tissue without the need for a tissue biopsy ${ }^{28}$. Suspected non-melanoma skin cancer, scar tissue and healthy skin were identified with a balanced accuracy of $95 \%$, highlighting the potential for Raman spectroscopy to guide tumour resection, allowing clean margins to be obtained in real time without the need for additional surgeries.

\section{Biofluids}

Despite the wealth of information provided via tissue and cell interrogation, one of the major drawbacks is the need for surgery or other invasive procedures to extract sample biopsies for diagnosis and prognosis information. In light of this, the use of biofluids in vibrational spectroscopy has seen numerous publications in recent years and provides a minimally invasive, rapid and label-free technique to analyse biological samples ${ }^{59-64}$. Biofluids, such as blood, urine and saliva, are particularly attractive for diagnostic studies as they are easily accessible ${ }^{63,65}$. 
In recent years advanced analytical techniques, in particular machine learning algorithms, are being utilised with spectroscopy due to their ability to recognise patterns and changes in the broad spectral fingerprint associated with disease ${ }^{66-73}$. An FTIR study of 389 patients with acute chest pain was shown to differentiate patient with acute myocardial infarction from those with minor chest pains of other origins, allowing therapeutic attention to be given to those in need of it most ${ }^{74}$. This IR transflection mode study led by Petrich et al. conceives a diagnostic pattern recognition method that has the potential to diagnose within the first 6 hours after the onset of chest pain. The spectral results were collated rapidly and were found to be comparable to routine cardiac laboratory markers on the same dataset.

The IR analysis of whole blood samples and their derivatives have been used for the detection of Hepatitis B and C viruses, human immunodeficiency virus (HIV) and malaria by a research group in Australia 47-49,75,76. In a study utilising blood films on glass slides and ATR-FTIR spectroscopy, the detection of the malaria parasite was detected with specificity and sensitivity of $98 \%$ and $70 \%$, respectively. Additionally, the concentrations of both glucose and urea were also detected. This is important as with the progression of the malaria disease, the demand for glucose by the virus is high and blood glucose levels tend to fall, causing hypoglycaemia. In severe causes of malaria, the virus can interfere with renal function resulting in high levels of urea in the blood compared with healthy controls, therefore testing urea levels can help determine progression of the disease. The use of ATR-FTIR here allows the rapid and simultaneous quantification of multiple analytes in a single measurement ${ }^{47}$. Similarly, the detection of Hepatitis $\mathrm{C}$ at varying levels of infection has been characterised using Raman spectroscopy of patient blood plasma samples ${ }^{77}$. Current diagnostic tests for this virus utilise polymerase chain reaction (PCR) or enzyme-linked immunosorbent assays (ELISA) which are costly, laborious methods and they tend to require specific reagents. The use of Raman spectroscopy offers a rapid and label-free method of diagnosis, detecting the biochemical changes associated with Hepatitis C.

Huntington's Disease (HD) is an incurable and inherited neurological disorder leading to progressive motor and cognitive decline. Despite many attempts for the diagnosis of HD using specific blood biomarkers, no single biomarker has shown to be reliable in the conclusive diagnosis or disease progression of HD. However, the use of Raman spectroscopy by Huefner et al. has allowed identification of HD patients from healthy controls using serum ${ }^{78}$. 
Additionally, the use of surface enhanced Raman spectroscopy (SERS) offered a higher degree of specificity when monitoring disease progression, which may enable diagnosis at very early stages.

Many proof-of-principle oncology studies using biofluids have established the use of IR spectroscopies for the detection of cancers and progression of the disease. For example, survival rates for brain cancers are low with a ten-year survival rate of only $15 \%$, which is primarily due to the non-specific symptoms and thus delay to diagnosis. As a result of this, most patients with brain cancer present as an emergency with scans often showing advanced progression of disease. We have previously demonstrated the capability of IR spectroscopy utilising ATR mode to diagnose brain cancer and further classify these brain tumours into subtypes ${ }^{79-83}$. Utilising serum samples from a total of 433 patients, of which 122 did not have cancer, successful classification of cancer versus non cancer was achieved with sensitivity and specificity as high as $97.1 \%$ and $95.1 \%$, respectively. Furthermore, the severity of tumour grade could also be identified with high sensitivities and specificities. A further study combining the SVM classification technique with ATR-FTIR spectroscopy has seen the identification of brain cancer versus non cancer on a larger study containing 724 patients. The results obtained an average sensitivity and specificity of $93.2 \%$ and $92 \%$ on a per patient basis ${ }^{82}$. Further study on this patient cohort tested and compared different machine learning algorithms combined with class balancing techniques, which all resulted in similar sensitivity and specificity values, highlighting the potential for biofluid spectroscopy to aid in distinguishing between brain tumour types ${ }^{81}$. Thus, the rapid diagnosis via serum spectroscopy holds advantageous over current clinical diagnostic techniques, as patients typically require multiple brain scans to determine the presence and severity of a brain tumour, which can be overwhelming for the patient and very costly to the health services.

Current biomarker detection technologies for early-stage ovarian cancer are ineffective and the disease can often go undetected until it has reached its later stages, sparking the need for alternative early detection methodologies. Through ATR-FTIR spectroscopy, patient plasma samples were diagnosed as cancerous or healthy with $96 \%$ of patients identified correctly ${ }^{84}$. Additional ATR-FTIR studies concluded that the ovarian cancer patient plasma samples could be separated into disease stage with high sensitivity and specificity $(>91 \%){ }^{68}$. A different study assessed the ability of classification algorithms combined with ATR-FTIR spectra of urine to diagnose patients with endometrial cancer or ovarian cancer from healthy controls ${ }^{85}$. 
Using SVM with principal component analysis (PCA) for feature selection, accuracies greater than $92 \%$ were obtained.

Backhaus et al. have demonstrated the use of transflection and transmission mode serum spectroscopy combined with cluster analysis and ANN analysis to distinguish from breast cancer patients and healthy controls. Current breast cancer diagnoses in clinics are often complicated, time consuming and subjective. The use of IR spectroscopy offers a rapid and simple means to diagnosis, and as described in this study extremely high sensitivities and specificities (>95\%) are attainable. Spectral comparisons with 11 other diseases, covering a total of 3119 patients, assured spectral changes observed were in fact from breast cancer ${ }^{86}$. Similarly, for gastric cancer the process of diagnosis is uncomfortable for patients and is often dependant on the judgement of the pathologist. However, Sheng et al. have shown the ability of FTIR spectroscopy to diagnose gastric cancer from healthy controls, by using a ratio of amplitudes provided by the stretching vibrations of RNA and DNA found in serum ${ }^{87}$. Despite testing a very small patient cohort the method achieved encouraging results, with 12 blind samples leading to a sensitivity of $85.7 \%$ and specificity of $100 \%$.

The minimal water signal detected via Raman spectroscopy makes it highly appropriate for biofluid analysis. Mahmood et al. have shown the ability of Raman spectroscopy combined with multivariate analysis in identifying patients infected by the dengue virus ${ }^{88}$. Distinct features in the Raman spectra have been assigned to different immunoglobulin proteins and have been able to successfully monitor disease progression. Results attained were comparable to the outcomes achieved using ELISA and PCR techniques, yet the spectroscopic method is much faster with lower associated costs. Using similar analytical techniques, Sahu et al. have predicted the recurrence of oral cancers from serum collected before and after surgery ${ }^{89}$. Average classification efficiencies of $81 \%$ and $82 \%$ using principal component discriminant analysis of patient serum samples collected before and after surgery, respectively. The ability to predict relapse would undoubtedly benefit patients.

Colorectal cancers have also been classified against control samples using patient serum, with a Raman-based approach coupled with PCA providing accuracies as high as $88 \%$. In the spectra from those with colorectal cancer, bands associated with tryptophan, phenylalanine and betacarotene were found to exhibit a lower Raman intensity ${ }^{90}$. This study demonstrates the 
advantage of spectroscopy, not only allowing detection of disease but also identifying specific blood-based biomarkers which could aid with the understanding of the disease.

Arguably one of the major advantages of spectroscopic analysis is the ability to measure multiple analytes in a single non-destructive measurement. Ultimately, gaining more information on a patient's health on a much faster timescale when compared to techniques currently in use in clinics (e.g. ELISA) is admirable. The detection and quantification of 8 major constituents of serum - total protein, glucose, cholesterol, high and low density lipoproteins, triglycerides, urea and uric acid - have been made from a study containing 247 patients by comparing Raman and IR spectroscopies ${ }^{91,92}$. In order to make these measurements in the current clinical environment, multiple measurements would be required, which would necessitate a significantly larger sample volume when compared with spectroscopic studies of biofluids. Not only do spectroscopic techniques require minute sample volumes, but also less sample preparation and they are not dependant on availability of reagents, thus have much lower associated costs.

Advances in technology have also enabled strides toward high-throughput analysis and large scale trials, making vibrational spectroscopy techniques more applicable for healthcare settings 82,93-95. The development of disposable internal reflection elements (IREs) for ATR-FTIR modalities combined with an automated slide indexing unit allows accurate movement of the IRE across the spectrometer for high-throughput analysis ${ }^{82}$. Additionally, robotically spotting of serum samples onto a 384 multi-well titre plates combined with automated FTIR has also been established allowing high-throughput spectroscopic analysis of serum ${ }^{95}$. As a result of the many proof-of-concept applications of vibrational spectroscopy for disease detection, suggested protocols for sample preparation and analysis of biological materials using FTIR spectroscopies have been published ${ }^{96,97}$. Despite the wealth of evidence from many studies around the world, there still appears to be various obstacles to overcome before it can be translated to a healthcare setting. 


\section{Barriers to Translation}

As already discussed, there has been an abundance of diagnostic studies in the field of biomedical vibrational spectroscopy in the last few decades signifying its potential clinical utility ${ }^{98-105}$. Although, the transition from research to routine clinical testing has faced several major technological challenges, as well as the challenges presented from current patient pathways and the persuasion of clinicians on the reliability of a new diagnostic test.

\section{Technical Challenges}

Due to a lack of standardisation, the majority of the variance in clinical data occurs within the pre-analytical phase. A recent study reported that most of the inconsistencies $(\sim 61 \%)$ are introduced prior to carrying out the analysis ${ }^{106}$. There are several pre-analytical steps that can affect sample integrity if they have not been performed correctly.

One problem with the spectroscopic analysis of tissue is that heterogeneity within the specimen is not unusual, where tissue sections contain both tumour and normal tissues or several different grades of tumour ${ }^{107}$. The best alternative to making in vivo measurements is the use of fresh tissue samples, however it can be difficult to acquire the necessary ethical approval as well as finding suitable volunteers, thus currently formalin fixation and paraffin embedded (FFPE) tissue is the preferred source for the histological examination of tissue sections. Use of FFPE tissues is admirable for early studies - for both Raman and FTIR analysis - mainly due to the ease of collection from biobanks and the ability to reanalyse if required, though it may not be directly comparable to fresh tissue since there can be contributions to the signal from some of the chemicals used in the FFPE and dewaxing (paraffin removal) processes ${ }^{108,109}$. The formalin or ethanol - used in the dewaxing process - induces coagulation of the globular proteins present in the cytoplasm, which can result in the loss of structural integrity of potentially important organelles. Furthermore, the use of ethanol can precipitate lipid molecules that are not preserved through the primary fixation step. Preparation of cells for spectral analysis can also encounter some technical challenges, mostly based on the chemical fixation and drying processes. Cells are naturally present in a hydrated form, whereby water molecules are bound to macromolecules which contributes to their structural integrity and function ${ }^{110}$. Many studies initially focused on an air-drying process to dehydrate the cells directly onto substrates, however the this can result in delocalization of biomolecules as a result of large surface tension forces associated with passing the water-air interface. Another fundamental aspect of sample preparation that can influence cellular biochemistry is the surface on which they are grown. 
The surface can induce changes in cell adhesion and motility, in their proliferation and differentiation and in gene expression ${ }^{110}$. To overcome the confounding factors of the drying process, the cells must be appropriately fixed by means of chemical fixation methods. Unfortunately, all of the currently employed fixation approaches can still affect the resulting Raman or FTIR spectra. The main vibrational bands that are known to be modified by the current methods of tissue and cell processing are outlined and summarised in Table I.

Table I - Summary of affected bands in Raman and FTIR spectroscopy through currently employed tissue and cell processing methods ${ }^{109-115}$.

\begin{tabular}{|c|c|c|c|}
\hline & & Raman bands affected & FTIR bands affected \\
\hline \multirow{3}{*}{$\begin{array}{c}\text { Tissue } \\
\text { processing }\end{array}$} & Cryopreservation & $\begin{array}{l}\text { Decreased intensity in the } 1002 \text {, } \\
1447 \text { and } 1637 \mathrm{~cm}^{-1} \text { bands }\end{array}$ & $\begin{array}{l}\text { Shifting of Amide I and II bands, and } \\
\text { reduction in overall intensity }\end{array}$ \\
\hline & Chemical fixation & $\begin{array}{l}\text { Change in appearance of } 1490 \mathrm{~cm}^{-1} \\
\text { and reduction in intensity of } \\
\text { Amide I }\end{array}$ & Shift in Amide I/II bands by $\sim 10 \mathrm{~cm}^{-1}$ \\
\hline & Paraffin embedding & $\begin{array}{l}\text { Paraffin signals at } \sim 1063, \sim 1296, \\
\sim 1441 \mathrm{~cm}^{-1} \text { and contributions from } \\
\text { xylene } \sim 1002,1032,1203 \mathrm{~cm}^{-1}\end{array}$ & $\begin{array}{l}\sim 1465 \mathrm{~cm}^{-1} \text { and bands between } 2850- \\
2950 \mathrm{~cm}^{-1} \text { altered }\end{array}$ \\
\hline \multirow{5}{*}{$\begin{array}{c}\text { Cell } \\
\text { Processing }\end{array}$} & Air drying/desiccation & $\begin{array}{l}\text { Loss of features between 1253- } \\
1127 \mathrm{~cm}^{-1} \text { and }\end{array}$ & - \\
\hline & $\begin{array}{l}\text { Formalin/critical } \\
\text { point drying }\end{array}$ & $\begin{array}{l}\text { Loss of features between } 1600- \\
1500 \text { and } 1448-1127 \mathrm{~cm}^{-1} \text {; various } \\
\text { protein, nucleic acid and lipid } \\
\text { bands affected }\end{array}$ & $\begin{array}{l}\text { Loss of ester lipid peak due to acetone } \\
\text { and ethanol }\left(\sim 1750 \mathrm{~cm}^{-1}\right)\end{array}$ \\
\hline & $\begin{array}{l}\text { Glutaraldehyde- } \\
\text { osmium tetroxide and } \\
\text { critical point drying }\end{array}$ & - & $\begin{array}{l}\text { Decreased intensity of } 1000 \mathrm{~cm}^{-1} \text { and } \\
\text { less resolved lipid band } \sim 1750 \mathrm{~cm}^{-1}\end{array}$ \\
\hline & $\begin{array}{l}\text { Methanol:acetic acid } \\
(3: 1)\end{array}$ & $\begin{array}{l}\text { Various nucleic acid }\left(<1000 \mathrm{~cm}^{-1},\right. \\
\left.1060-1095 \mathrm{~cm}^{-1}\right) \text {, protein }(1668- \\
\left.1661,1450,1339-1305,1176 \mathrm{~cm}^{-1}\right) \\
\text { and lipid } 1447-1453,1339-1247, \\
\sim 1065 \mathrm{~cm}-1) \text { bands disturbed }\end{array}$ & - \\
\hline & Acetone & - & $\begin{array}{l}\text { Diminished cellular lipid bands } \\
\left(1740,2925 \mathrm{~cm}^{-1}\right) \text { and alterations to } \\
\text { the Amide I/II bands }\end{array}$ \\
\hline
\end{tabular}

With regards to biofluid analysis, the quality of a blood sample can be distorted by variables that occur during the blood collection; fragile veins, insufficient sample mixing and interruption of blood flow during collection can all result in the sample clotting, or activation of the coagulation cascade which could lead to abnormal clotting results ${ }^{116,117}$. Biospecimens must be stored in mechanical freezers at extremely low temperatures to prevent degradation and the destruction of important biomolecules, yet the temperature and duration of time that blood samples are stored are currently not standardised ${ }^{118-121}$. The number of freeze-thaw 
cycles should also be minimised, in order to avoid alterations in protein concentrations and potential discrepancies in clinical tests ${ }^{122}$. The integrity of a blood sample can also be compromised by molecular contamination through fractionation methods, which in turn can affect the spectroscopic signature ${ }^{123,124}$. Sample preparation can also influence the reproducibility of spectral data, as well as intra- and inter-operator variation. Standardising the preparation of biofluids is not a simple task, as the analysed sample state will be dependent on the available equipment and/or the project requirements. When analysing biofluids through FTIR spectroscopy, liquid drops are often dehydrated onto a substrate due to the spectral interference of water ${ }^{125-128}$. On the other hand, aqueous samples are well suited to Raman spectroscopy since water is a weak Raman scatterer, hence it is less common for biofluids to be dehydrated prior to Raman measurements ${ }^{129}$. We have published various studies in the last decade exploring these types of pre-analytical factors that seem to inhibit the clinical progression of FTIR spectroscopy, as well as computational methods to overcome some of those barriers ${ }^{130-134}$. To date, there are no pre-analytical research studies published in the literature relating to a Raman-based approach, yet this may be a requirement for clinical Raman spectroscopy to reach its full potential.

There are certain analytical and post-analytical factors that can cause issues, albeit less common. The operation of instruments from various manufacturers can occasionally cause discrepancies, making it difficult to reliably compare and contrast spectra from different spectrometers. For spectroscopic analysis of tissue, we have technical limitations like lengthy acquisition times for imaging; signal detection; the difficulty of making quantitative measurements of tissue composition in vivo, and ambient light effects, as well as several considerations in data handling - such as spectral pre-processing, feature extraction and tissue classification ${ }^{135}$. Likewise, other analytical issues can stem from altering the collection parameters, such as the sampling mode, number of scans and spectral resolution. Similarly, post-analytical variation may be attributed to the plethora of available data analysis packages and computational analysis software, containing several types of basic statistical analysis, multivariate analysis and machine learning algorithms. Research groups can utilise various types of software and analysis to process their data in order to achieve the best results for their dataset, thus the output and statistics may not be directly comparable even within the same field of expertise. 
An interesting overview of factors slowing down progression of clinical applications of FTIR imaging was described by Goormaghtigh et al., which focused on the variation in pre-analytical conditions, spectrometer settings and collection parameters ${ }^{136}$. Furthermore, some recommendations on these spectral histopathology imaging parameters - such as tissue section thickness, air purging, pixel size and data pre-processing - were suggested by the authors to try and drive further discussion on the topic, and ultimately determine a final consensus for routine clinical analyses of tissue specimens.

A later publication from Santos and colleagues reviewed the actual clinical needs in oncology that could potentially be addressed by Raman spectroscopy ${ }^{137}$. Yet again, the concluding remarks emphasised that most of the applications addressed in proof-of-principle studies are still far from clinical adoption and commercialisation. The need for the consistency of protocols is evident, and has been nicely put by Dey et al. in their recent review article, as without standardisation they state that: "we are comparing apples to oranges and may be stuck with numbers that are not comparable from one research group to the other, thus leading nowhere" 138 .

\section{Clinical Issues}

There are also several clinical issues that must be considered. Defining the clinical question and unmet need is generally the first step in translational research for the treatment or diagnosis of a disease or clinical problem ${ }^{139}$. The unmet need should be fully explored, and the diagnostic pathway must be carefully mapped out in order to understand the issues that need to be resolved 140. An important aspect of clinical research is the close interaction between clinicians and experts in the field ${ }^{141}$. Coupling this clinical question to an appropriate technique or technology is the next consideration. This may require technical developments in order to best approach and suit the clinical need, with automation often being a desirable feature of any new intervention $^{142}$.

Another common issue is that many proof-of-concept studies involve relatively small patient cohorts, and this can over-estimate the significance of the results as they are not entirely representative of larger populations ${ }^{143}$. Sample numbers are often a limiting factor in the development of new technologies since the recruitment of sample specimens from patients through clinical studies can often be difficult and take several months or years, leading to many studies with limited cohorts. Even when some funding is secured for the purchasing of 
biospecimens, biobanks and tissue banks may not have the preferred disease and/or control samples in order to conduct the study - for example, due to the scarcity of a particular disease/demographics data making many samples unsuitable for the purpose of the study. A noticeable pattern in the literature is an alarming low number of patient numbers used to establish early utility, which can often pose questions on the reliability of the results. The use of machine learning algorithms to classify between patient sets is heavily influenced by the number of subjects fed into the training and testing of these computational models ${ }^{144}$. Despite the availability of various sampling methods that can overcome class imbalance in training and testing sets ${ }^{145}$, they often struggle with small datasets.

Likewise, the majority of these studies are based on retrospective samples, and these cohorts can be susceptible to bias as the disease states of the patients are already known. Hence, when progressing on to a prospective study, it is common to observe a lesser diagnostic performance 146. These effects are commonly described as 'spectrum bias', which accounts for the variation in test performance for prediction, screening and disease diagnostics among different population subgroups. For example, a test that has been developed in a population with higher disease prevalence, will normally have a lower sensitivity and higher specificity when applied in a population with lower prevalence of disease ${ }^{147}$. Likewise, symptomatic patients who do not have the disease become more prevalent in prospective studies, so it can be more difficult to successfully detect the disease in these cohorts. Thus, determining the true clinical utility may only be possible through studies conducted in the targeted clinical environment.

Furthermore, there is not yet a widely recognised threshold for the statistical performance for diagnostic tests - in terms of the minimum accuracy, sensitivity, specificity, area under the curve (AUC) for receiving operating characteristic (ROC) curves etc. - that would be an acceptable clinical value. Likewise, it is unclear which parameter between sensitivity and specificity is preferred to assess the diagnostic potential. For example, several studies endeavour to maximise sensitivity $(>90 \%)$ by sacrificing specificity $(\sim 50 \%)$, and conversely, others appear to tailor their machine learning algorithms to enhance specificity, which in turn can reduce the sensitivity. There is also inconsistency with how patient training and testing population splits are determined then reported. Instead of ensuring the spectral data from one individual patient cannot be in both the training and testing datasets, some studies have spectra from one patient in both sets, which can overestimate their diagnostic ability ${ }^{148}$. Similarly, there should be several train/test splits by utilising resampling methods to guarantee different 
patients are being tested on, yet it is not uncommon only to see only one train/test split, with the results being reported from single classification models. Moreover, the proportion of patients in the testing set can be variable across studies, e.g. a 70:30 split (70\% training and $30 \%$ testing) or a 60:40 split. These factors may be dependent on the desired application, but a more standardised approach would undoubtedly enable a more efficient clinical translation.

\section{Health Economic and Regulatory Barriers}

In addition to scientific and clinical development, there is also the commercial element. Intellectual property (IP) applications, difficulty in securing funding and international markets can often represent a further barrier to translation. There is often a lack of concrete understanding of what the unmet clinical needs are, and the limitations of the current gold standard techniques ${ }^{10}$. As already discussed, the standardisation of sample preparation and measurement protocols is inadequate, and with a wide array of data pre-processing and analysis techniques to choose from, the lack of consistency in the vibrational spectroscopy field could be detrimental for potential funding and inhibit progress ${ }^{149}$. There are many challenges that new technologies face when trying to protect their IP. Patent applications are very expensive and will often face renewal fees. Currently, there is no single patent that covers IP worldwide, thus it is difficult to protect IP in the multiple jurisdictions around the world, and the lack of a more universal patent system leaves small innovating companies in fear of potential global competitors ${ }^{150}$. The development of a start-up or spin-out company may appear to be the ideal pathway to commercialisation ${ }^{151,152}$. This can be a daunting task for many researchers; hence it is crucial to seek business advice and discover the approach to successful IP strategies ${ }^{153}$.

There are currently two major hurdles placed in the path to successful translation; 1) approval from regulatory agencies for marketing and 2) the acceptance from health technology assessment (HTA) agencies who determine which technologies are made available in the public health system. Regulatory agencies are responsible for ensuring new medicines and medical devices are safe, such as the Food and Drug Administration (FDA) in the USA, the European Medicines Agency (EMA) in the EU, and the Medicines and Healthcare products Regulatory Agency (MHRA) in the UK. These agencies ensure the manufacturer of new interventions follow the appropriate conformity assessment procedure, which is essential for products to attain CE marking and comply with relevant health, safety and environmental requirements ${ }^{154}$. Criteria for successful acceptance require statistically verified clinical trials to prove medical 
utility, but also clear understanding of the current diagnostic pathways in order to determine the economic and clinical impact of new technologies. For successful translation of such techniques, it is vital to examine the existing competition, define where in the current pathway the proposed 'diagnostic test' would be best suited, and undertake an economic evaluation to establish the potential cost and health benefits for the health services.

Health economics concerns how the health services allocates its funds ${ }^{155}$. Health economists commonly undertake an economic evaluation, which analyses alternative courses of action - in terms of both their costs and consequences - often in comparison to current practices ${ }^{156,157}$. Health economists will often carry out a cost-effectiveness analysis (CEA), which is a comparison of costs in monetary units with outcomes in quantitative non-monetary terms, with respect to the best currently available treatment, strategy or intervention ${ }^{158}$. The benefits of an intervention can be portrayed by variety of different outcomes, such as the differences in quality adjusted life years (QALY) incorporates the impact on both the quantity and quality of life of a proposed intervention ${ }^{159}$. In the UK, the National Institute for Health and Care Excellence (NICE) make decisions regarding whether a new technology will be funded within the UK's National Health Service (NHS), and this is informed by the evidence of costeffectiveness. Economic evaluation and CEA have been progressively employed in the last few decades to examine the feasibility of novel pharmaceuticals, drugs, biomarkers and medical devices ${ }^{160}$. Notably, the first health economic assessment (HEA) of a new technology relating to vibrational spectroscopy was issued recently in the BMJ Open. In 2018, Gray et al. published a pre-trial HEA for the introduction of a serum-based spectroscopic blood test into the clinic 161. The proposed test was for the diagnosis of brain tumours, which utilises ATR-FTIR spectroscopy to detect the biosignature of the disease from a patient's blood serum ${ }^{162,163}$. The current pathway for brain tumour diagnosis consists of magnetic resonance imaging (MRI) or computed tomography (CT) imaging followed by surgical biopsy and histopathology. MRI and CT imaging are considerably expensive: a single patient costs the NHS around $\sim £ 300$ for imaging alone ${ }^{161}$. A reliable blood serum test at the primary care setting could fast track these patients into the diagnostic pathway much quicker, whilst reducing the number of unnecessary brain scans, saving the health services on avoidable spending. A further economic evaluation recently looked at updating this early economic model with the results from a prospective clinical diagnostic accuracy study ${ }^{164}$, and also explored the effect of brain tumour type predictions on the patient outcomes and healthcare costs. The authors concluded even after the 
inclusion of the prospective clinical data, the technology still has the potential to be costeffective in both primary and secondary care ${ }^{165}$.

This is only one example of a health economic assessment for a new spectroscopic intervention, but there is a real lack of CEAs in the literature that relate to the clinical translation of spectroscopic techniques for other applications. These types of analyses are essential before proof-of-concept studies can progress into the next phase of determining their clinical utility. Ultimately, biomedical vibrational spectroscopists who are eager to successfully translate their research must consider the following: the unmet needs of the current diagnostic pathway/their target application; how their results compare to the current gold standard methods; the costing of their new technology or intervention; and the benefits to the health services and/or population. 


\section{Where we are now?}

\section{Raman}

Vibrational spectroscopy has been at the forefront of translational clinical research for many years now. In 2015, Stevens et al. reviewed the development of fibre optic Raman probe instrumentation for clinical applications ${ }^{166}$. Numerous factors were considered for the potential translation into clinical workflow ensuring materials and design were compatible for the target outcomes. This review concluded that in vivo clinical diagnostics, surgical targeting and treatment monitoring using Raman probe technologies could be the way forward for numerous clinical applications ${ }^{166}$. In the same year, a Raman-based approach utilising a handheld probe for live, local detection of cancer cells in the human brain was established by Jermyn et al. (Figure 4) ${ }^{167}$. Using this probe intra-operatively, the authors successfully differentiated normal brain from dense cancer and normal brain invaded by cancer cells. This Raman-based probe enabled detection of the previously undetectable diffusely invasive brain cancer cells in various glioma grades. The authors later published a similar in situ intra-operative approach, with a more generic cancer detection system that combines intrinsic fluorescence spectroscopy, diffuse reflectance spectroscopy, and Raman spectroscopy ${ }^{168}$. Using this multimodal optical cancer detection system, Jermyn et al. highlighted brain, lung, colon, and skin cancers could be detected in situ during surgery with an accuracy, sensitivity, and specificity of $97 \%, 100 \%$, and $93 \%$, respectively. A more recent study by Livermore et al. demonstrated the ability of Raman spectroscopy to aid neurosurgeons in determining personalised treatment during surgery, by targeting specific glioma subtypes in situ ${ }^{169}$. Knowledge on the genetic subtype of the patient's glioma at the time of surgery would allow the surgeon to tailor their strategy for the best possible prognosis. These types of intra-operative technologies have the potential to classify cell populations in real time, making it an ideal guide for surgical resection and decision-making.

$<$ Insert Figure 4 here $>$ 
An article by Krafft et al. focused on developments with coherent Raman scattering (CRS) imaging techniques outlining the need for translation to hospitals while discussing the unmet needs in biomedicine, clinical diagnosis and healthcare applications ${ }^{170}$. The paper also outlined the extensive amount of literature within the field of CRS microscopy over the last twenty years and the need for networks such as the International Society for Clinical Spectroscopy (CLIRSPEC) and Raman4Clinics in order to push the clinical translation through collaborative efforts. Pence and Mahadevan-Jansen continued the discussion of utilising Raman spectroscopy for clinical applications, citing several modalities of Raman scattering, including: Raman imaging, high wavenumber region (HWN) Raman, spatially offset Raman spectroscopy (SORS) as well as surface enhanced Raman spectroscopy (SERS) ${ }^{171}$. They also noted that the most common clinical target is cancer with all research groups fundamentally having the same goals: i) to improve early detection with high sensitivity and specificity, ii) understanding/monitoring the disease processes and iii) easy access to the target organ being as non-invasive to the patient as possible.

More specific research involves the use of HWN Raman spectroscopy to investigate the diagnostic capabilities of tumours by comparison of water, protein and lipid content in surrounding healthy tissue. Although this idea was displayed by Garcia-Flores et al. back in 2001 by identifying differences in water signals for breast tumours in rat models ${ }^{172}$, it has only recently been adapted to explore human breast tissue combined with fibre optic probes for intra-operative margin analysis (IMA) ${ }^{173}$. Similarly, Puppels' research group in the Netherlands - who had previously published distinctions between oral cavity squamous cell carcinoma (OCSCC) and healthy tissue using HWN Raman spectroscopy ${ }^{174}$ - recently developed a fibre optic needle probe to improve the accuracy of specimen-driven intraoperative assessment of resection margins (IOARM) ${ }^{175}$. Other recent literature of particular interest includes publications by Avram et al. who developed a liquid biopsy for diagnosing colorectal cancer with SERS ${ }^{176}$, Nicolson et al. who reported the first in vivo study of surface enhanced SORS for brain cancer imaging ${ }^{177}$, and Du et al. who reported a serum-based Raman spectroscopy analysis of the hepatitis B virus ${ }^{178}$.

The translation from academic research to the clinic environment requires collaborations between researchers, clinicians, industrial partners, and funding bodies. Currently there are numerous national and international research initiatives surrounding Raman spectroscopy 
techniques, including the aforementioned CLIRSPEC and Raman4Clinics, as well as the Coherent Raman Imaging for the Molecular Study of the Origin of Diseases (CRIMSON).

CRIMSON involves collaborative efforts from numerous research groups, partners and enterprises ${ }^{179}$. It is funded through the European Union's Horizon 2020 research and innovation programme and lasts from December 2020 for 42 months. The primary aim of CRIMSON is to understand cellular origin of diseases by the development of a vibrational spectroscopy-based cell/tissue imaging system. The focus of the project involves CRS combined with microscope/endoscope for inter- and intra-cellular processes. The European consortium involves collaborative efforts from research centres including Politecnico di Milano (Italy), Leibniz Institute of Photonic Technology e.V and IF-CNRS (Germany); biomedical partners such as Istituto Nazionale dei Tumori (Italy), Institut National de la Santé Et de la Recherche Médicale (France) and Jena University Hospital (Germany); as well as small and medium-sized enterprises, Active Fiber Systems GmbH (Germany), Lightcore Technologies (France), Cambridge Raman Imaging Ltd (UK) and 3rdPlace (Italy) ${ }^{180}$. Through this trans-disciplinary collaboration CRIMSON aims to provide solutions to numerous limitations currently found with CRS applications, including the ability to detect within the fingerprint region, use of multiple vibrational frequencies and the development of a low-cost, compact laser system. Validation of the new imaging system will focus on cancer with a clear cellular origin, targeting autophagic processes, interactions between cancer and immune cells, and senescence ${ }^{179}$.

The Raman Nanotheranostics (RaNT) research programme is currently developing a novel allin-one technology to diagnose and treat cancers with a single device ${ }^{181}$. The project is led by Prof. Nick Stone at the University of Exeter, collaborating with scientists at the University of Cambridge, the UCL School of Pharmacy and the Science and Technology Facilities Council Rutherford Appleton Laboratories, all based in the UK. The Engineering and Physical Sciences Research Council (EPSRC) awarded RaNT a £5.7 million grant in 2018 for a 5-year period, and the team is focused on reducing the need for cancer surgery by providing rapid, pain-free detection and therapy, right at the patient bedside.

Companies such as RiverD International B.V. (Netherlands) provide commercially available Raman spectroscopy instrumentation, and they are specifically targeted towards quantitative in vivo skin analysis ${ }^{182}$. However, they do also supply custom-designed microscope accessories 
for near-IR Raman microspectroscopy. The skin analysis can be widely utilised for occupational skin exposure, trans-dermal drug delivery monitoring and also developing, optimising and testing topical products ${ }^{183}$. The accessories available to purchase can provide countless opportunities for analysis of cells and tissues using near-infrared Raman spectroscopy for diagnostic applications.

Two collaborative large-scale cross-laboratory studies have recently been published by members of Raman4Clinics, off the back of the COST action plan ${ }^{184,185}$. In the study by Guo et al., Raman spectral data from 15 different institutes was contrasted and compared to determine potential intra- and inter-operability ${ }^{184}$. The authors concluded by recommending a number of future actions, such as a standardised spectrometer calibration approach for manufacturers, development of SOPs for instrument verification, calibration and performance, and encouraging researchers to make their data and findings openly available to establish larger databases.

\section{Infrared}

There have also been several exciting developments within the IR clinical spectroscopy community and the technique shows great promise for translation into diverse healthcare pathways. For biofluid applications, ClinSpec Diagnostics Ltd. (ClinSpec Dx) recently demonstrated the clinical potential of a disposable silicon ATR-FTIR test for serum-based cancer diagnostics, with discrimination of brain cancer and non-cancer control samples with sensitivity and specificity of $93.2 \%$ and $92.8 \%$ respectively in a retrospective study ${ }^{81,186}$. ClinSpec Dx further participated in a prospective clinical validation study comprised of 385 blinded patients where the technology showed high diagnostic capability in a clinical environment, with sensitivity and specificity of $81 \%$ and $80 \%$ respectively ${ }^{164}$. As already discussed, the overwhelming majority of research efforts have reported clinical test performance based on retrospective patient studies. The transition to prospective studies is an important step towards the realisation of IR tests in the clinic, as it provides technological validation within clinical pathways and establishes analytical confidence with clinical end users. Ultimately, the execution of the initial health economic evaluation by Gray et al. was instrumental in defining key test parameters in the research and development phase of the technology, as well as demonstrating a strong economic argument for clinical adoption to healthcare decision-makers and potential investors ${ }^{161}$. Thus far, few health economic evaluations have been published on IR technologies despite the plethora of promising clinical 
research. These studies must be strongly considered by the field going forward to initiate conversations with key decision-makers and health technology assessment agencies.

Biotech Resources, developed by Professor Wood's and Dr Heraud's research team in collaboration with the Monash University Centre for Biospectroscopy, is another emblematic example of how IR technologies are on the cusp of clinical adoption for biofluid diagnostics. Biotech Resources have developed a low-cost, simple point-of-care test based on ATR-FTIR, for analysis of blood and its derivatives for a diverse range of clinical test applications. Biotech Resources have shown significant clinical potential of their technology for malaria diagnostics ${ }^{187-190}$, and have recently conducted feasibility studies in real clinical environments in Papa New Guinea and Thailand ${ }^{191}$. Recent test results of 318 suspected malaria patients at four regional clinics in Thailand demonstrated high test sensitivity and specificity of $92 \%$ and $97 \%$ respectively ${ }^{188}$. The point-of-care system, which analysed spectroscopic measurements on a cloud-based system, and subsequently delivered clinical test results to a mobile phone or computer, highlights the accessibility of the technology for mass screening of malaria in resource limited countries. As well as detection of parasites, they have shown the clinical utility of their spectroscopic approach for identification of bacterial and fungal pathogens ${ }^{192,193}$, and hepatitis viruses ${ }^{75}$. In fact, Biotech Resources have recently been awarded an investment from the Australian Government's \$35 million BioMedTech Horizons program, for development of a rapid spectroscopic test for direct detection of bacterial and fungal pathogens that cause sepsis in patients from whole blood ${ }^{194}$. Additionally, the spin-out company are developing a rapid antibiotic susceptibility test capable of detecting resistance to numerous antibiotics in under two hours from positive blood cultures ${ }^{195}$. Ultimately, the identification of parasites, viruses, bacteria and fungi is enabled by detection of specific biochemical signatures that correspond to distinct vibrational modes associated with nucleic acid, lipid and protein constituents in blood, and highlights the breadth of possibilities of IR spectroscopy for clinical biofluid diagnostics in the future.

Recent commercialisation of IR spectroscopy for disease diagnostics is further evident from developments at Glyconics, who are an IR diagnostics company in the UK developing proprietary handheld FTIR devices for analysis of diverse samples to enable point-of-care diagnostics for multiple conditions ${ }^{196}$. Initially, they wish to target chronic obstructive pulmonary disease (COPD) and diabetes mellitus. Recently, they completed a licensing agreement with Ghent University (Belgium) for worldwide exclusivity and future collaboration 
on IR spectroscopy technologies that include determination of protein glycation in fingernails for diagnosis and continued monitoring of diabetes mellitus ${ }^{197-199}$. Very recently, Glyconics were also recipient of a UK Research and Innovation grant as part of the Global Challenges Research Fund, to study the clinical feasibility of implementing IR technologies for diabetic screening in developing countries, which re-iterates the clinical potential of IR spectroscopy for resource limited settings.

The clinical potential of IR spectroscopy further extends to analysis of biological tissue and represents an attractive future alternative to histopathology for cancer diagnostics in clinical laboratories. The projection of IR light onto tissue sections has several analytical benefits and would permit simultaneous characterisation of different biomolecules in a non-destructive, low-cost manner without the need for expensive and time-consuming staining and labelling procedures ${ }^{200}$. Furthermore, the acquisition of spectroscopic information would allow for a complete biochemical fingerprint of tissue samples, as oppose to exclusively identifying tissue architecture and protein biomarkers with antibodies using immunohistopathology ${ }^{201}$. Thus far, spectral histopathology has demonstrated considerable diagnostic potential for numerous malignancies, including lung, colorectal adenoma, melanoma and breast cancers 202-206. However, one aspect of spectral histopathology that has previously restricted clinical translation concerns the limited acquisition time to scan entire tissue samples.

Typically, spectral histopathology utilises focal plane array detectors that may take several hours to days to complete scanning of an individual tissue sample and requires significant computational capabilities to store tens of thousands of collected spectra ${ }^{207}$. This is undoubtedly an advancement on previous single point mode analysis with single element detectors, but still has significantly reduced sample throughput compared to conventional histopathology and is not conducive to busy clinical laboratories. Nevertheless, recent technological advances and different sampling formats have emerged that make spectral histopathology more attractive for routine clinical adoption. The emergence of quantum cascade lasers (QCL's) represents an important advancement for clinical translation of spectral histopathology and permits measurement of discrete frequencies pertinent to biological discrimination between sample types, rather than measuring all wavenumbers of an entire IR spectrum ${ }^{208,209}$. This has significant implications for acquisition time, allowing tissue samples to be analysed within minutes to hours ${ }^{210}$. Recent adoption of tissue micro-arrays (TMA's) in spectral histopathology further extends time advantages compared to previous approaches of 
fixing tissue sections to calcium or barium fluoride substrates, or low emissivity slides. The high-brilliance of QCL's permits spectroscopic interrogation with increased signal-to-noise and diffraction limited resolution, which enables improved analysis of sub-cellular details in tissue for enhanced diagnostic capabilities ${ }^{211}$. Lastly, adoption of room-temperature QCL's negates the need for cooling with liquid nitrogen and allows simplification of instrumental design, which undoubtedly makes spectral histopathology more attractive for clinical translation in future years ${ }^{212,213}$.

The recent adoption of these new approaches for spectral histopathology and their subsequent integration with conventional histopathology instrumentation is perhaps best underlined through the seminal work of the Bhargava group, based in Illinois ${ }^{214}$. The authors recently demonstrated an all-digital, 'stain-less' staining hybrid platform that combined wide-field visible microscopy with a QCL IR light source and tissue micro-arrays for analysis of breast cancer tissue. The study highlighted that the integration of IR spectroscopy and machine learning with ubiquitous optical microscopy offered the possibility of label-free molecular characterisation of tissue in an easy to use, accessible format within clinical laboratories, and further underlines the importance of designing IR technologies that are suitable and compatible with current clinical workflows.

$<$ Insert Figure 5 here $>$

\section{Conclusion}

It is clear that both Raman and IR spectroscopy hold great promise for diverse clinical applications and there now needs to be a concerted effort alongside clinicians, instrument developers, commercial enterprises, health economists and healthcare decision makers to ensure the technology reaches its full potential and translates to the clinic. Typically, commercial spectroscopic and imaging systems are built for research laboratories and not for medical diagnostics, thus many of the currently available analytical instruments are not necessarily compatible with clinical workflows. Many proof-of-concept studies are performed within academia on a 3-4 years' timescale which is not realistic when standardisation of protocols, methodologies, validations and translation requires a much greater timescale. The 
question of "How good is good enough?", is still discussed within the community however the numerous on-going collaborations between researchers, clinicians and enterprises provides hope for translation of spectroscopy into the clinical environment within the coming years ${ }^{10,149}$.

In order to further aid translation and realisation of patient benefit we need to revisit and update the requirements suggested by many researchers over the years. Whilst it should be noted that with time and progress of the field these propositions may change, it is the opinion of the authors that to overcome the initial barriers to translation, we should be focusing on:

1) A standardised protocol for samples preparation or further studies on the pre-analytical factors that affect a particular sample preparation.

2) Performing research projects and studies with sufficient sample numbers and appropriate statistical analysis techniques so valid conclusions can be drawn. If a study only has a small number of samples in each disease and/or healthy group, then there needs to be a valid reason provided for this and a recognition of the impact upon results reported by the authors of the study. As reviewers of current peer-reviewed papers we can uphold this.

3) Results should be reported correctly and the requirements for reporting should be standardised. This can be something the field decides upon in the future, but a good starting point would be to review and begin to include the requirements of the Standard for Reporting of Diagnostic Accuracy Studies (STARD) statement ${ }^{215}$ (long-standing approach to accurate reporting of diagnostics studies and as such has been accepted by multiple journals and researchers alike).

4) Show evidence that the level of performance has some economic benefit to the area, within which the authors are aiming to translate the research to. An understanding of the limitations of the proposed test / clinical environment in terms of how the test would be used, what the workflow would be and the overall economics of what is required. This would start to focus studies on the correct applications. Of course, there are many examples of research that have not had a target application or appreciation of this that have made a huge impact in medicine, but in order to maximise the probability of success, we feel that this is necessary.

5) Start to prepare for, and engage in, large scale trials of the technology in order to truly show clinical effectiveness. This must be proven in a symptomatic population with correct symptomatic controls in order to establish accurate measures of sensitivity and specificity, highlighting the potential issues of comorbidities. 


\section{References}

1. M. Jackson, M.G. Sowa, H.H. Mantsch. "Infrared spectroscopy: a new frontier in medicine". Biophys Chem. 1997. 68(1-3): 109-125. 10.1016/s0301-4622(97)80555-8.

2. "Advantages and Disadvantages of MRI". Basics of Magnetic Resonance Imaging. Springer US, Boston, MA, 1988. Pp. 125-138. 10.1007/978-1-4613-2081-4 9.

3. R.R. Edelman. "The History of MR Imaging as Seen through the Pages of Radiology". Radiology. 2014. 273(2S): S181-S200. 10.1148/radiol.14140706.

4. R. Damadian. "Tumor Detection by Nuclear Magnetic Resonance". Science. 1971. 171(3976): 1151-1153. 10.1126/science.171.3976.1151.

5. $\quad$ P.C. Lauterbur. "Image Formation by Induced Local Interactions: Examples Employing Nuclear Magnetic Resonance". Nature. 1973. 242(5394): 190-191. 10.1038/242190a0.

6. American Physical Society. "MRI Uses Fundamental Physcis for Clinical Diagnosis". APS Physics News. 2006.

https://www.aps.org/publications/apsnews/200607/history.cfm\#: :text=On\%20July\%203\%2C \%201977\%2C\%20the,bodily\%20structures\%20and\%20diagnosing\%20disease. [Feb 1 2021].

7. Nobel Media AB. The Nobel Prize in Physiology or Medicine 2003. 2003.

8. M. Jackson. "Introductory Lecture”. Faraday Disc. 2004. 126: 1. 10.1039/b313625f.

9. W. Petrich. "Translating vibrational spectroscopy into clinical applications - vision or reality?" Faraday Discuss. 2016. 187: 603-607. 10.1039/C6FD00108D.

10. H.J. Byrne, M. Baranska, G.J. Puppels, N. Stone, B. Wood, K.M. Gough, et al. "Spectropathology for the next generation: Quo vadis?" The Analyst. 2015. 140(7): 20662073. 10.1039/C4AN02036G.

11. CLIRSPEC. "The International Society for Clinical Spectroscopy (CLIRSPEC)". n.d. https://clirspec.org/ [Feb 3 2021].

12. Raman4Clinics. "Raman4Clinics". n.d. https://www.raman4clinics.eu/ [Feb 3 2021].

13. European Cooperation, in the field of Scientific, and Technical Research. COST Action BM1401, EUROPEAN NETWORK ON RAMAN-BASED APPLICATIONS FOR CLINICAL DIAGNOSTICS (Raman4clinics). 2014.

14. Z.S. Morris, S. Wooding, J. Grant. "The answer is 17 years, what is the question: understanding time lags in translational research". Journal of the Royal Society of Medicine. 2011. 104(12): 510-520. 10.1258/jrsm.2011.110180.

15. S.R. Hanney, S. Castle-Clarke, J. Grant, S. Guthrie, C. Henshall, J. Mestre-Ferrandiz, et al. "How long does biomedical research take? Studying the time taken between biomedical and health research and its translation into products, policy, and practice". Health Res Policy Sys. 2015. 13(1): 1. 10.1186/1478-4505-13-1.

16. D.I. Ellis, R. Goodacre. "Metabolic fingerprinting in disease diagnosis: Biomedical applications of infrared and Raman spectroscopy". Analyst. 2006. 131(8): 875-885. $10.1039 / \mathrm{b} 602376 \mathrm{~m}$.

17. N. Grinberg, S. Rodriguez, eds. Ewing's Analytical Instrumentation Handbook. 4th ed. CRC Press, Boca Raton, FL, 2019.

18. D.R. Parachalil, B. Brankin, J. McIntyre, H.J. Byrne. "Raman spectroscopic analysis of high molecular weight proteins in solution-considerations for sample analysis and data preprocessing". Analyst. Royal Society of Chemistry, 2018. 143(24): 5987-5998. 10.1039/c8an01701h.

19. R. Pieper, C.L. Gatlin, A.J. Makusky, P.S. Russo, C.R. Schatz, S.S. Miller, et al. "The human serum proteome: Display of nearly 3700 chromatographically separated protein spots on twodimensional electrophoresis gels and identification of 325 distinct proteins". Proteomics. 2003. 3: 1345-1364. 10.1002/pmic.200300449.

20. S. Hu, J. Loo, D. Wong. Human body fluid proteome analysis. Proteomics. 2006. 6326-6353. 10.1002/pmic.200600284.

21. T.D. Veenstra, T.P. Conrads, B.L. Hood, A.M. Avellino, R.G. Ellenbogen, R.S. Morrison. "Biomarkers: Mining the Biofluid Proteome *". Molecular and Cellular Proteomics. 2005. 4.4: 409-418. 10.1074/mcp.M500006-MCP200. 
22. L.B. Leal, M.S. Nogueira, R.A. Canevari, L.F.C.S. Carvalho. "Vibration spectroscopy and body biofluids: Literature review for clinical applications". Photodiagnosis and Photodynamic Therapy. Elsevier, 2018. 24: 237-244. 10.1016/j.pdpdt.2018.09.008.

23. C. Krafft, V. Sergo. "Biomedical applications of Raman and infrared spectroscopy to diagnose tissues". Spectroscopy. 2006. 20: 195-218. 10.1155/2006/738186.

24. K. Isensee, N. Kröger-Lui, W. Petrich. "Biomedical applications of mid-infrared quantum cascade lasers-a review". Analyst. Royal Society of Chemistry, 2018. 143(24): 5888-5911. $10.1039 / \mathrm{c} 8 \mathrm{an} 01306 \mathrm{c}$.

25. M.J. Baker, H.J. Byrne, J. Chalmers, P. Gardner, R. Goodacre, A. Henderson, et al. "Clinical applications of infrared and Raman spectroscopy: state of play and future challenges". The Analyst. Royal Society of Chemistry, 2018. 143: 1735-1757. 10.1039/C7AN01871A.

26. O. Uckermann, R. Galli, S. Tamosaityte, E. Leipnitz, K.D. Geiger, G. Schackert, et al. "Labelfree delineation of brain tumors by coherent anti-Stokes Raman scattering microscopy in an orthotopic mouse model and human glioblastoma". PLoS ONE. 2014. 9(9).

10.1371/journal.pone.0107115.

27. J. Nallala, M.D. Diebold, C. Gobinet, O. Bouché, G.D. Sockalingum, O. Piot, et al. "Infrared spectral histopathology for cancer diagnosis: A novel approach for automated pattern recognition of colon adenocarcinoma". Analyst. 2014. 139(16): 4005-4015. 10.1039/c3an01022h.

28. C.A. Lieber, S.K. Majumder, D.L. Ellis, D.D. Billheimer, A. Mahadevan-Jansen. "In vivo nonmelanoma skin cancer diagnosis using Raman microspectroscopy". Lasers in Surgery and Medicine. 2008. 40(7): 461-467. 10.1002/1sm.20653.

29. F.M. Lyng, I.R.M. Ramos, O. Ibrahim, H.J. Byrne. "Vibrational microspectroscopy for cancer screening". Applied Sciences (Switzerland). 2015. 5(1): 23-35. 10.3390/app5010023.

30. K. Kong, C. Kendall, N. Stone, I. Notingher. "Raman spectroscopy for medical diagnostics From in-vitro biofluid assays to in-vivo cancer detection". Advanced Drug Delivery Reviews. The Authors, 2015. 89: 121-134. 10.1016/j.addr.2015.03.009.

31. A. Nijssen, S. Koljenovic, T.C.B. Schut, P.J. Caspers, G.J. Puppels. "Towards oncological application of Raman spectroscopy”. Journal of Biophotonics. 2009. 2(1): 29-36. 10.1002/jbio.200810055.

32. H.J. Byrne, F. Bonnier, Z. Farhane. "Two-dimensional correlation analysis of Raman microspectroscopy of subcellular interactions of drugs in vitro". Journal of Biophotonics. 2019. 12(3): 1-10. 10.1002/jbio.201800328.

33. Z. Movasaghi, S. Rehman, I.U. Rehman. "Raman spectroscopy of biological tissues". Applied Spectroscopy Reviews. 2007. 42(5): 493-541. 10.1080/05704920701551530.

34. P. Lasch, D. Naumann. "Spatial resolution in infrared microspectroscopic imaging of tissues". Biochimica et Biophysica Acta - Biomembranes. 2006. 1758(7): 814-829. 10.1016/j.bbamem.2006.06.008.

35. K. Gajjar, L.D. Heppenstall, W. Pang, K.M. Ashton, J. Trevisan, I.I. Patel, et al. "Diagnostic segregation of human brain tumours using Fourier-transform infrared and/or Raman spectroscopy coupled with discriminant analysis". Analytical Methods. 2013. 5: 89-102. $10.1039 / \mathrm{c} 2 \mathrm{ay} 25544 \mathrm{~h}$.

36. N.C. Purandare, J. Trevisan, I.I. Patel, K. Gajjar, A.L. Mitchell, G. Theophilou, et al. "Exploiting biospectroscopy as a novel screening tool for cervical cancer: towards a framework to validate its accuracy in a routine clinical setting". Bioanalysis. 2013. 5(21): 2697-2711. 10.4155/bio.13.233.

37. I. Behl, G. Calado, O. Ibrahim, A. Malkin, S. Flint, H.J. Byrne, et al. "Development of methodology for Raman microspectroscopic analysis of oral exfoliated cells". Anal. Methods. 2017. 9(6): 937-948. 10.1039/C6AY03360A.

38. K.M. Ostrowska, A. Malkin, A. Meade, J. O'Leary, C. Martin, C. Spillane, et al. "Investigation of the influence of high-risk human papillomavirus on the biochemical composition of cervical cancer cells using vibrational spectroscopy". Analyst. 2010. 135(12): 3087.

10.1039/c0an00571a. 
39. G.E. Menzies, H.R. Fox, C. Marnane, L. Pope, V. Prabhu, S. Winter, et al. "Fourier transform infrared for noninvasive optical diagnosis of oral, oropharyngeal, and laryngeal cancer".

Translational Research. 2014. 163(1): 19-26. 10.1016/j.trsl.2013.09.006.

40. P.D. Lewis, K.E. Lewis, R. Ghosal, S. Bayliss, A.J. Lloyd, J. Wills, et al. "Evaluation of FTIR spectroscopy as a diagnostic tool for lung cancer using sputum". BMC Cancer. 2010. 10: 640650 .

41. B. Bird, M. Miljkovi, S. Remiszewski, A. Akalin, M. Kon, M. Diem. "Infrared spectral histopathology (SHP): A novel diagnostic tool for the accurate classification of lung cancer". Laboratory Investigation. 2012. 92(9): 1358-1373. 10.1038/labinvest.2012.101.

42. J. Sule-Suso, D. Skingsley, G.D. Sockalingum, A. Kohler, G. Kegelaer, M. Manfait, et al. "FTIR microspectroscopy as a tool to assess lung cancer cells response to chemotherapy". Vibrational Spectroscopy. 2005. 38: 179-184. 10.1016/j.vibspec.2005.02.010.

43. C.L. Song, M.Z. Vardaki, R.D. Goldin, S.G. Kazarian. "Fourier transform infrared spectroscopic imaging of colon tissues: evaluating the significance of amide $\mathrm{I}$ and $\mathrm{C}-\mathrm{H}$ stretching bands in diagnostic applications with machine learning”. Analytical and Bioanalytical Chemistry. Analytical and Bioanalytical Chemistry, 2019. 411(26): 6969-6981. 10.1007/s00216-019-02069-6.

44. K. Araki, N. Yagi, Y. Ikemoto, H. Yagi, C.J. Choong, H. Hayakawa, et al. "Synchrotron FTIR micro-spectroscopy for structural analysis of Lewy bodies in the brain of Parkinson's disease patients". Scientific Reports. Nature Publishing Group, 2015. 5(November): 1-8. $10.1038 /$ srep 17625 .

45. J. Vongsvivut, D. Pérez-Guaita, B.R. Wood, P. Heraud, K. Khambatta, D. Hartnell, et al. "Synchrotron macro ATR-FTIR microspectroscopy for high-resolution chemical mapping of single cells". Analyst. Royal Society of Chemistry, 2019. 144(10): 3226-3238. $10.1039 / \mathrm{c} 8 \mathrm{an} 01543 \mathrm{k}$.

46. V. Balan, C.T. Mihai, F.D. Cojocaru, C.M. Uritu, G. Dodi, D. Botezat, et al. "Vibrational spectroscopy fingerprinting in medicine: From molecular to clinical practice". Materials. 2019. 12(18): 1-40. 10.3390/ma12182884.

47. S. Roy, D. Perez-Guaita, D.W. Andrew, J.S. Richards, D. McNaughton, P. Heraud, et al. "Simultaneous ATR-FTIR Based Determination of Malaria Parasitemia, Glucose and Urea in Whole Blood Dried onto a Glass Slide". Analytical Chemistry. 2017. 89(10): 5238-5245. 10.1021/acs.analchem.6b04578.

48. P. Heraud, P. Chatchawal, M. Wongwattanakul, P. Tippayawat, C. Doerig, P. Jearanaikoon, et al. "Infrared spectroscopy coupled to cloud-based data management as a tool to diagnose malaria: A pilot study in a malaria-endemic country". Malaria Journal. BioMed Central, 2019. 18(1): 1-11. 10.1186/s12936-019-2945-1.

49. M. Martin, D. Perez-Guaita, D.W. Andrew, J.S. Richards, B.R. Wood, P. Heraud. "The effect of common anticoagulants in detection and quantification of malaria parasitemia in human red blood cells by ATR-FTIR spectroscopy". Analyst. Royal Society of Chemistry, 2017. 142(8): 1192-1199. 10.1039/c6an02075e.

50. A. Khoshmanesh, M.W.A. Dixon, S. Kenny, L. Tilley, D. McNaughton, B.R. Wood. "Detection and quantification of early-stage malaria parasites in laboratory infected erythrocytes by attenuated total reflectance infrared spectroscopy and multivariate analysis". Analytical Chemistry. 2014. 86(9): 4379-4386. 10.1021/ac500199x.

51. T.J.E. Hubbard, A. Shore, N. Stone. "Raman spectroscopy for rapid intra-operative margin analysis of surgically excised tumour specimens". Analyst. Royal Society of Chemistry, 2019. 144(22): 6479-6496. 10.1039/c9an01163c.

52. J.N. Bentley, M. Ji, X.S. Xie, D.A. Orringer. "Real-time image guidance for brain tumor surgery through stimulated Raman scattering microscopy". Expert Review of Anticancer Therapy. 2014. 14(4): 359-361. 10.1586/14737140.2013.877844.

53. E. Cordero, J. Rüger, D. Marti, A.S. Mondol, T. Hasselager, K. Mogensen, et al. "Bladder tissue characterization using probe-based Raman spectroscopy: Evaluation of tissue heterogeneity and influence on the model prediction". Journal of Biophotonics. 2020. 13(2): 114. $10.1002 /$ jbio.201960025. 
54. K. Kong, F. Zaabar, E. Rakha, I. Ellis, A. Koloydenko, I. Notingher. "Towards intra-operative diagnosis of tumours during breast conserving surgery by selective-sampling Raman microspectroscopy". Phys. Med. Biol. 2014. 59(20): 6141-6152. 10.1088/0031-9155/59/20/6141.

55. J. Desroches, M. Jermyn, M. Pinto, F. Picot, M.A. Tremblay, S. Obaid, et al. "A new method using Raman spectroscopy for in vivo targeted brain cancer tissue biopsy". Scientific Reports. 2018. 8(1): 1-10. 10.1038/s41598-018-20233-3.

56. M. Ji, D.A. Orringer, C.W. Freudiger, S. Ramkissoon, X. Liu, D. Lau, et al. "Rapid, label-free detection of brain tumours with stimulated Raman scattering microscopy". Science Translational Medicine. 2014. 5(201). 10.1126/scitranslmed.3005954.Rapid.

57. L.F. Santos, R. Wolthuis, S. Koljenović, R.M. Almeida, G.J. Puppels. "Fiber-optic probes for in vivo Raman spectroscopy in the high-wavenumber region". Analytical Chemistry. 2005. 77(20): 6747-6752. 10.1021/ac0505730.

58. J. Desroches, M. Jermyn, K. Mok, C. Lemieux-Leduc, J. Mercier, K. St-Arnaud, et al. "Characterization of a Raman spectroscopy probe system for intraoperative brain tissue classification". Biomedical Optics Express. 2015. 6(7): 2380. 10.1364/boe.6.002380.

59. A. Bonifacio, S. Cervo, V. Sergo. "Label-free surface-enhanced Raman spectroscopy of biofluids : fundamental aspects and diagnostic applications". Analytical and Bioanalytical Chemistry. 2015. 407: 8265-8277. 10.1007/s00216-015-8697-z.

60. J. Ollesch, S.L. Drees, H.M. Heise, T. Behrens, T. Brüning, K. Gerwert. "FTIR spectroscopy of biofluids revisited: an automated approach to spectral biomarker identification". Analyst. 2013. 138: 4092-4102. 10.1039/c3an00337j.

61. A.L. Mitchell, K.B. Gajjar, G. Theophilou, F.L. Martin, P.L. Martin-Hirsch. "Vibrational spectroscopy of biofluids for disease screening or diagnosis: Translation from the laboratory to a clinical setting". Journal of Biophotonics. 2014. 7(3-4): 153-165. 10.1002/jbio.201400018.

62. A. Rohman, A. Windarsih, E. Lukitaningsih, M. Rafi, K. Betania, N.A. Fadzillah. "The use of FTIR and Raman spectroscopy in combination with chemometrics for analysis of biomolecules in biomedical fluids: A review". Biomedical Spectroscopy and Imaging. 2019. 8: 55-71. 10.3233/BSI-200189.

63. M.J. Baker, G.D. Sockalingum, C. Hughes, R.A. Lukaszewski. "Developing and Understanding Biofluid Vibrational Spectroscopy: a Critical Review". Chemical Society Reviews. 2016. 45: 1803-1818. 10.1039/c5cs00585j.

64. K. Kochan, D.E. Bedolla, D. Perez-Guaita, J.A. Adegoke, T. Chakkumpulakkal Puthan Veettil, M. Martin, et al. "Infrared Spectroscopy of Blood". Appl Spectrosc. 2021. 000370282098585. $10.1177 / 0003702820985856$.

65. M. Paraskevaidi, P.L. Martin-Hirsch, F.L. Martin. "ATR-FTIR spectroscopy tools for medical diagnosis and disease investigation". Nanotechnology Characterization Tools for Biosensing and Medical Diagnosis. Springer, 2018. Pp. 163-211. 10.1007/978-3-662-56333-5_4.

66. B.R. Smith, M.J. Baker, D.S. Palmer. "PRFFECT: A versatile tool for spectroscopists". Chemometrics and Intelligent Laboratory Systems. Elsevier Ltd, 2018. 172(October 2017): 33-42. 10.1016/j.chemolab.2017.10.024.

67. L. Lovergne, P. Bouzy, V. Untereiner, R. Garnotel, M.J. Baker, G. Thiéfin, et al. "Biofluid infrared spectro-diagnostics: pre-analytical considerations for clinical applications". Faraday Discussions. 2016. 187: 521-537. 10.1039/C5FD00184F.

68. K.M.G. Lima, K.B. Gajjar, P.L. Martin-Hirsch, F.L. Martin. "Segregation of ovarian cancer stage exploiting spectral biomarkers derived from blood plasma or serum analysis: ATR-FTIR spectroscopy coupled with variable selection methods". Biotechnology Progress. 2015. 31(3): 832-839. 10.1002/btpr.2084.

69. L. Sitole, F. Steffens, T.P.J. Krüger, D. Meyer. "Mid-ATR-FTIR spectroscopic profiling of HIV/AIDS sera for novel systems diagnostics in global health". OMICS A Journal of Integrative Biology. 2014. 18(8): 513-523. 10.1089/omi.2013.0157.

70. D.K.R. Medipally, D. Cullen, V. Untereiner, G.D. Sockalingum, A. Maguire, T.N.Q. Nguyen, et al. "Vibrational spectroscopy of liquid biopsies for prostate cancer diagnosis". Therapeutic Advances in Medical Oncology. 2020. 12: 1-23. 10.1177/1758835920918499. 
71. S.S.S.J. Ahmed, W. Santosh, S. Kumar, T.H. Thanka Christlet. "Neural network algorithm for the early detection of Parkinson's disease from blood plasma by FTIR micro-spectroscopy". Vibrational Spectroscopy. 2010. 53: 181-188. 10.1016/j.vibspec.2010.01.019.

72. S.L. Haas, R. Müller, A. Fernandes, K. Dzeyk-Boycheva, S. Würl, J. Hohmann, et al. "Spectroscopic diagnosis of myocardial infarction and heart failure by fourier transform infrared spectroscopy in serum samples". Applied Spectroscopy. 2010. 64: 262-267. 10.1366/000370210790918508.

73. A. Lux, R. Müller, M. Tulk, C. Olivieri, R. Zarrabeita, T. Salonikios, et al. "HHT diagnosis by Mid-infrared spectroscopy and artificial neural network analysis". Orphanet Journal of Rare Diseases. 2013. 8: 1-15. 10.1186/1750-1172-8-94.

74. W. Petrich, K.B. Lewandrowski, J.B. Muhlestein, M.E.H. Hammond, J.L. Januzzi, E.L. Lewandrowski, et al. "Potential of mid-infrared spectroscopy to aid the triage of patients with acute chest pain". Analyst. 2009. 134(6): 1092-1098. 10.1039/b820923e.

75. S. Roy, D. Perez-Guaita, S. Bowden, P. Heraud, B.R. Wood. "Spectroscopy goes viral: Diagnosis of hepatitis B and C virus infection from human sera using ATR-FTIR spectroscopy”. Clinical Spectroscopy. Elsevier B.V., 2019. 1(January): 100001. 10.1016/j.clispe.2020.100001.

76. C. Pizarro, I. Esteban-Díez, I. Arenzana-Rámila, J.M. González-Sáiz. "Discrimination of patients with different serological evolution of HIV and co-infection with HCV using metabolic fingerprinting based on Fourier transform infrared". Journal of Biophotonics. 2018. 11(3): 1-12. 10.1002/jbio.201700035.

77. A. Ditta, H. Nawaz, T. Mahmood, M.I. Majeed, M. Tahir, N. Rashid, et al. "Principal components analysis of Raman spectral data for screening of Hepatitis C infection". Spectrochimica Acta - Part A: Molecular and Biomolecular Spectroscopy. 2019. 221. 10.1016/j.saa.2019.117173.

78. A. Huefner, W.L. Kuan, S.L. Mason, S. Mahajan, R.A. Barker. "Serum Raman spectroscopy as a diagnostic tool in patients with Huntington's disease". Chemical Science. Royal Society of Chemistry, 2020. 11(2): 525-533. 10.1039/c9sc03711j.

79. J.R. Hands, G. Clemens, R. Stables, K. Ashton, A. Brodbelt, C. Davis, et al. "Brain tumour differentiation: rapid stratified serum diagnostics via attenuated total reflection Fouriertransform infrared spectroscopy". Journal of Neuro-Oncology. Springer US, 2016. 127: 463472. 10.1007/s11060-016-2060-x.

80. J.R. Hands, K.M. Dorling, P. Abel, K.M. Ashton, A. Brodbelt, C. Davis, et al. "Attenuated Total Reflection Fourier Transform Infrared (ATR-FTIR) spectral discrimination of brain tumour severity from serum samples". Journal of Biophotonics. 2014. 7: 189-199. 10.1002/jbio.201300149.

81. J.M. Cameron, H.J. Butler, B.R. Smith, M.G. Hegarty, M.D. Jenkinson, K. Syed, et al. "Developing infrared spectroscopic detection for stratifying brain tumour patients: glioblastoma multiforme vs . lymphoma". Analyst. Royal Society of Chemistry, 2019. 144: 6736-6750. 10.1039/c9an01731c.

82. H.J. Butler, P.M. Brennan, J.M. Cameron, D. Finlayson, M.G. Hegarty, M.D. Jenkinson, et al. "Development of high-throughput ATR-FTIR technology for rapid triage of brain cancer". Nature Communications. Springer US, 2019. 10: 4501. 10.1038/s41467-019-12527-5.

83. J.M. Cameron, C. Rinaldi, H.J. Butler, M.G. Hegarty, P.M. Brennan, M.D. Jenkinson, et al. "Stratifying Brain Tumour Histological Sub-Types: The Application of ATR-FTIR Serum Spectroscopy in Secondary Care". Cancers. 2020. 12(7): 1710. 10.3390/cancers12071710.

84. K. Gajjar, J. Trevisan, G. Owens, P.J. Keating, N.J. Wood, H.F. Stringfellow, et al. "Fouriertransform infrared spectroscopy coupled with a classification machine for the analysis of blood plasma or serum: A novel diagnostic approach for ovarian cancer". Analyst. 2013. 138(14): 3917-3926. 10.1039/c3an36654e.

85. M. Paraskevaidi, C.L.M. Morais, K.M.G. Lima, K.M. Ashton, H.F. Stringfellow, P.L. MartinHirsch, et al. "Potential of mid-infrared spectroscopy as a non-invasive diagnostic test in urine for endometrial or ovarian cancer". Analyst. Royal Society of Chemistry, 2018. 143(13):

3156-3163. 10.1039/c8an00027a. 
86. J. Backhaus, R. Mueller, N. Formanski, N. Szlama, H.-G. Meerpohl, M. Eidt, et al. "Vibrational Spectroscopy Diagnosis of breast cancer with infrared spectroscopy from serum samples". Vibrational Spectroscopy. 2010. 52: 173-177. 10.1016/j.vibspec.2010.01.013.

87. D. Sheng, Y. Wu, X. Wang, D. Huang, X. Chen, X. Liu. "Comparison of serum from gastric cancer patients and from healthy persons using FTIR spectroscopy". Spectrochimica Acta Part A: Molecular and Biomolecular Spectroscopy. Elsevier B.V., 2013. 116: 365-369. 10.1016/j.saa.2013.07.055.

88. T. Mahmood, H. Nawaz, A. Ditta, M.I. Majeed, M.A. Hanif, N. Rashid, et al. "Raman spectral analysis for rapid screening of dengue infection". Spectrochimica Acta - Part A: Molecular and Biomolecular Spectroscopy. Elsevier B.V., 2018. 200: 136-142. 10.1016/j.saa.2018.04.018.

89. A. Sahu, N. Nandakumar, C.M. Krishna. "Recurrence prediction in oral cancers: a serum Raman spectroscopy study". Analyst. Royal Society of Chemistry, 2015. 140: 2294-2301. 10.1039/c4an01860e.

90. X. Li, T. Yang, S. Li. "Discrimination of serum Raman spectroscopy between normal and colorectal cancer using selected parameters and regression-discriminant analysis". Applied Optics. 2012. 51: 5038-5043. 10.1364/AO.51.005038.

91. D. Rohleder, W. Kiefer, W. Petrich. "Quantitative analysis of serum and serum ultrafiltrate by means of Raman spectroscopy”. Analyst. 2004. 129(10): 906-911. 10.1039/b408927h.

92. D. Rohleder, G. Kocherscheidt, K. Gerber, W. Kiefer, W. Köhler, J. Möcks, et al. "Comparison of mid-infrared and Raman spectroscopy in the quantitative analysis of serum". Journal of Biomedical Optics. 2005. 10(3): 031108. 10.1117/1.1911847.

93. C. Hughes, G. Clemens, B. Bird, T. Dawson, K.M. Ashton, M.D. Jenkinson, et al. "Introducing Discrete Frequency Infrared Technology for High-Throughput Biofluid Screening". Scientific Reports. Nature Publishing Group, 2016. 6: 20173. 10.1038/srep20173.

94. C.A. Jenkins, R.A. Jenkins, M.M. Pryse, K.A. Welsby, M. Jitsumura, C.A. Thornton, et al. “A high-throughput serum Raman spectroscopy platform and methodology for colorectal cancer diagnostics". Analyst. 2018. 143(24): 6014-6024. 10.1039/c8an01323c.

95. J. Ollesch, M. Heinze, H.M. Heise, T. Behrens, T. Brüning, K. Gerwert. "It's in your blood: Spectral biomarker candidates for urinary bladder cancer from automated FTIR spectroscopy". Journal of Biophotonics. 2014. 7(3-4): 210-221. 10.1002/jbio.201300163.

96. M.J. Baker, J. Trevisan, P. Bassan, R. Bhargava, H.J. Butler, K.M. Dorling, et al. "Using Fourier transform IR spectroscopy to analyze biological materials". Nature Protocols. 2014. 9: 1771-1791. 10.1038/nprot.2014.110.

97. A.G. Theakstone, C. Rinaldi, H.J. Butler, J.M. Cameron, L.R. Confield, S.H. Rutherford, et al. "Fourier-transform infrared spectroscopy of biofluids: A practical approach". Translational Biophotonics. 2021. 10.1002/tbio.202000025.

98. R. Dukor. "Vibrational spectroscopy in the detection of cancer". Handbook of Vibrational Spectroscopy. J. M. Chalmers and P.R. Griffths Edition. Wiley, Chichester, 2002.

99. R.A. Shaw, S. Low-Ying, A. Man, K.-Z. Liu, C. Mansfield, C.B. Rileg, et al. "Infrared Spectroscopy of Biofluids in Clinical Chemistry and Medical Diagnostics". Biomedical Vibrational Spectroscopy. Hoboken, NJ: John Wiley and Sons, Inc. 2008. 79-103.

100. G. Bellisola, C. Sorio. "Infrared spectroscopy and microscopy in cancer research and diagnosis". American journal of cancer research. 2012. 2(1): 1.

101. H.J. Byrne. "Vibrational Spectroscopy: Disease Diagnostics and Beyond". Optical spectroscopy and computational methods in biology and medicine. Springer, New York, 2013.

102. A.L. Mitchell, K.B. Gajjar, G. Theophilou, F.L. Martin, P.L. Martin-Hirsch. "Vibrational spectroscopy of biofluids for disease screening or diagnosis: translation from the laboratory to a clinical setting: Vibrational spectroscopy of biofluids: laboratory to clinical setting". Journal of Biophotonics. 2014. 7(3-4): 153-165. 10.1002/jbio.201400018.

103. K. Kong, C. Kendall, N. Stone, I. Notingher. "Raman spectroscopy for medical diagnostics From in-vitro biofluid assays to in-vivo cancer detection". Advanced Drug Delivery Reviews. 2015. 89: 121-134. 10.1016/j.addr.2015.03.009.

104. M.J. Baker, C.S. Hughes, K.A. Hollywood. Biophotonics: vibrational spectroscopic diagnostics. Morgan \& Claypool, San Rafael, 2016. 
105. S.G. Kazarian. "Perspectives on infrared spectroscopic imaging from cancer diagnostics to process analysis". Spectrochimica Acta Part A: Molecular and Biomolecular Spectroscopy. 2021. 251: 119413. 10.1016/j.saa.2020.119413.

106. P. Carraro, M. Plebani. "Errors in a Stat Laboratory: Types and Frequencies 10 Years Later". Clinical Chemistry. 2007. 53(7): 1338-1342. 10.1373/clinchem.2007.088344.

107. D. Sebiskveradze, V. Vrabie, C. Gobinet, A. Durlach, P. Bernard, E. Ly, et al. "Automation of an algorithm based on fuzzy clustering for analyzing tumoral heterogeneity in human skin carcinoma tissue sections". Lab Invest. 2011. 91(5): 799-811. 10.1038/labinvest.2011.13.

108. C.M. Krishna, G.D. Sockalingum, L. Venteo, R.A. Bhat, P. Kushtagi, M. Pluot, et al. "Evaluation of the suitability of ex vivo handled ovarian tissues for optical diagnosis by Raman microspectroscopy”. Biopolymers. 2005. 79(5): 269-276. 10.1002/bip.20346.

109. C.W. Meuse, P.E. Barker. "Quantitative Infrared Spectroscopy of Formalin-fixed, Paraffinembedded Tissue Specimens: Paraffin Wax Removal With Organic Solvents". Applied Immunohistochemistry \& Molecular Morphology. 2009. 17(6): 547-552. 10.1097/PAI.0b013e3181a9300e.

110. F. Lyng, E. Gazi, P. Gardner. "Chapter 5. Preparation of Tissues and Cells for Infrared and Raman Spectroscopy and Imaging”. In: D. Moss, editor. RSC Analytical Spectroscopy Series. Royal Society of Chemistry, Cambridge, 2010. Pp. 145-191. 10.1039/9781849731997-00145.

111. E. Ó Faoláin, M.B. Hunter, J.M. Byrne, P. Kelehan, M. McNamara, H.J. Byrne, et al. "A study examining the effects of tissue processing on human tissue sections using vibrational spectroscopy". Vibrational Spectroscopy. 2005. 38(1-2): 121-127. 10.1016/j.vibspec.2005.02.013.

112. R. Wiens, M. Rak, N. Cox, S. Abraham, B.H.J. Juurlink, W.M. Kulyk, et al. "Synchrotron FTIR microspectroscopic analysis of the effects of anti-inflammatory therapeutics on wound healing in laminectomized rats". Anal Bioanal Chem. 2007. 387(5): 1679-1689. 10.1007/s00216-006-1095-9.

113. A.D. Meade, C. Clarke, F. Draux, G.D. Sockalingum, M. Manfait, F.M. Lyng, et al. "Studies of chemical fixation effects in human cell lines using Raman microspectroscopy". Anal Bioanal Chem. 2010. 396(5): 1781-1791. 10.1007/s00216-009-3411-7.

114. M.M. Mariani, P. Lampen, J. Popp, B.R. Wood, V. Deckert. "Impact of fixation on in vitro cell culture lines monitored with Raman spectroscopy". Analyst. 2009. 134(6): 1154. $10.1039 / \mathrm{b} 822408 \mathrm{k}$.

115. V. Untereiner, O. Piot, M.-D. Diebold, O. Bouché, E. Scaglia, M. Manfait. "Optical diagnosis of peritoneal metastases by infrared microscopic imaging”. Anal Bioanal Chem. 2009. 393(67): 1619-1627. 10.1007/s00216-009-2630-2.

116. N. Dhingra, Safe Injection Global Network, World Health Organization. WHO guidelines on drawing blood: best practices in phlebotomy. 2010.

117. S. Nicholas. Pre-Analytical Variables in the Clinical Chemistry Lab. Select Science. 2017.

118. L.-H. Huang, P.-H. Lin, K.-W. Tsai, L.-J. Wang, Y.-H. Huang, H.-C. Kuo, et al. "The effects of storage temperature and duration of blood samples on DNA and RNA qualities". R.N. PENA i SUBIRÀ, editor. PLoS ONE. 2017. 12(9): e0184692. 10.1371/journal.pone.0184692.

119. A. Hubel, R. Spindler, A.P.N. Skubitz. "Storage of Human Biospecimens: Selection of the Optimal Storage Temperature". Biopreservation and Biobanking. 2014. 12(3): 165-175. 10.1089/bio.2013.0084.

120. J.B. Vaught, M.K. Henderson. "Biological sample collection, processing, storage and information management". IARC Sci. Publ. 2011. (163): 23-42.

121. S.S. Tworoger. "Collection, Processing, and Storage of Biological Samples in Epidemiologic Studies: Sex Hormones, Carotenoids, Inflammatory Markers, and Proteomics as Examples". Cancer Epidemiology Biomarkers \& Prevention. 2006. 15(9): 1578-1581. 10.1158/10559965.EPI-06-0629.

122. J.-E. Lee, S.Y. Kim, S.-Y. Shin. "Effect of Repeated Freezing and Thawing on Biomarker Stability in Plasma and Serum Samples". Osong Public Health and Research Perspectives. 2015. 6(6): 357-362. 10.1016/j.phrp.2015.11.005.

123. F. Bonnier, F. Petitjean, M.J. Baker, H.J. Byrne. "Improved protocols for vibrational spectroscopic analysis of body fluids: Improved protocols for vibrational spectroscopic 
analysis of body fluids". Journal of Biophotonics. 2014. 7(3-4): 167-179. 10.1002/jbio.201300130.

124. F. Bonnier, M.J. Baker, H.J. Byrne. "Vibrational spectroscopic analysis of body fluids: avoiding molecular contamination using centrifugal filtration". Analytical Methods. 2014. 6(14): 5155. 10.1039/c4ay00891j.

125. R.D. Deegan. "Pattern formation in drying drops". Physical review E. 2000. 61(1): 475.

126. H. Hu, R.G. Larson. "Marangoni Effect Reverses Coffee-Ring Depositions". The Journal of Physical Chemistry B. 2006. 110(14): 7090-7094. 10.1021/jp0609232.

127. C. Hughes, M. Brown, G. Clemens, A. Henderson, G. Monjardez, N.W. Clarke, et al. "Assessing the challenges of FTIR spectroscopic analysis of blood serum". Journal of Biophotonics. 2014. 7(3-4): 180-188. 10.1002/jbio.201300167.

128. J.M. Cameron, H.J. Butler, D.S. Palmer, M.J. Baker. "Biofluid spectroscopic disease diagnostics: A review on the processes and spectral impact of drying". Journal of Biophotonics. 2018. 11(4): e201700299. 10.1002/jbio.201700299.

129. J.M. Cameron, C. Bruno, D.R. Parachalil, M.J. Baker, F. Bonnier, H.J. Butler, et al. "Vibrational spectroscopic analysis and quantification of proteins in human blood plasma and serum". Vibrational Spectroscopy in Protein Research. Elsevier, 2020. Pp. 269-314. 10.1016/B978-0-12-818610-7.00010-4.

130. L. Lovergne, G. Clemens, V. Untereiner, R.A. Lukaszweski, G.D. Sockalingum, M.J. Baker. "Investigating optimum sample preparation for infrared spectroscopic serum diagnostics". Anal. Methods. 2015. 7(17): 7140-7149. 10.1039/C5AY00502G.

131. L. Lovergne, P. Bouzy, V. Untereiner, R. Garnotel, M.J. Baker, G. Thiéfin, et al. "Biofluid infrared spectro-diagnostics: pre-analytical considerations for clinical applications". Faraday Discuss. 2016. 187: 521-537. 10.1039/C5FD00184F.

132. L. Lovergne, J. Lovergne, P. Bouzy, V. Untereiner, M. Offroy, R. Garnotel, et al. "Investigating pre-analytical requirements for serum and plasma based infrared spectrodiagnostic". J. Biophotonics. 2019. 12(12). 10.1002/jbio.201900177.

133. J.M. Cameron, H.J. Butler, D.J. Anderson, L. Christie, L. Confield, K.E. Spalding, et al. "Exploring pre-analytical factors for the optimisation of serum diagnostics: Progressing the clinical utility of ATR-FTIR spectroscopy". Vibrational Spectroscopy. 2020. 109: 103092. 10.1016/j.vibspec.2020.103092.

134. A. Sala, K.E. Spalding, K.M. Ashton, R. Board, H.J. Butler, T.P. Dawson, et al. "Rapid analysis of disease state in liquid human serum combining infrared spectroscopy and 'digital drying"'. J. Biophotonics. 2020. 13(9). 10.1002/jbio.202000118.

135. M. Jermyn, J. Desroches, K. Aubertin, K. St-Arnaud, W.-J. Madore, E. De Montigny, et al. “A review of Raman spectroscopy advances with an emphasis on clinical translation challenges in oncology". Phys. Med. Biol. 2016. 61(23): R370-R400. 10.1088/0031-9155/61/23/R370.

136. E. Goormaghtigh. "Infrared imaging in histopathology: Is a unified approach possible?" BSI. 2017. 5(4): 325-346. 10.3233/BSI-160151.

137. I.P. Santos, E.M. Barroso, T.C. Bakker Schut, P.J. Caspers, C.G.F. van Lanschot, D.-H. Choi, et al. "Raman spectroscopy for cancer detection and cancer surgery guidance: translation to the clinics". Analyst. 2017. 142(17): 3025-3047. 10.1039/C7AN00957G.

138. P. Dey, I. Blakey, N. Stone. "Diagnostic prospects and preclinical development of optical technologies using gold nanostructure contrast agents to boost endogenous tissue contrast". Chem. Sci. 2020. 11(33): 8671-8685. 10.1039/D0SC01926G.

139. A. Sala, D.J. Anderson, P.M. Brennan, H.J. Butler, J.M. Cameron, M.D. Jenkinson, et al. "Biofluid Diagnostics by FTIR Spectroscopy: A Platform Technology for Cancer Detection". Cancer Letters. 2020. S0304383520300835. 10.1016/j.canlet.2020.02.020.

140. E. Badrick, K. Cresswell, P. Ellis, A.G. Renehan, E.J. Crosbie, P. Crosbie, et al. "Top ten research priorities for detecting cancer early". The Lancet Public Health. 2019. 4(11): e551. 10.1016/S2468-2667(19)30185-9.

141. D. Finlayson, C. Rinaldi, M.J. Baker. "Is Infrared Spectroscopy Ready for the Clinic?" Anal. Chem. 2019. 91(19): 12117-12128. 10.1021/acs.analchem.9b02280. 
142. C. Kendall, M. Isabelle, F. Bazant-Hegemark, J. Hutchings, L. Orr, J. Babrah, et al. "Vibrational spectroscopy: a clinical tool for cancer diagnostics". Analyst. 2009. 134(6): 1029. 10.1039/b822130h.

143. A. Hackshaw. "Small studies: strengths and limitations". European Respiratory Journal. 2008. 32(5): 1141-1143. 10.1183/09031936.00136408.

144. H.M. Kalayeh, D.A. Landgrebe. "Predicting the Required Number of Training Samples". IEEE Trans. Pattern Anal. Mach. Intell. 1983. PAMI-5(6): 664-667. 10.1109/TPAMI.1983.4767459.

145. N.V. Chawla, K.W. Bowyer, L.O. Hall, W.P. Kegelmeyer. "SMOTE: Synthetic Minority Over-sampling Technique". Journal of Artificial Intelligence Research. 2002. 16: 321-357. 10.1613/jair.953.

146. J.W. Song, K.C. Chung. "Observational Studies: Cohort and Case-Control Studies": Plastic and Reconstructive Surgery. 2010. 126(6): 2234-2242. 10.1097/PRS.0b013e3181f44abc.

147. J.A. Usher-Smith, S.J. Sharp, S.J. Griffin. "The spectrum effect in tests for risk prediction, screening, and diagnosis". BMJ. 2016. i3139. 10.1136/bmj.i3139.

148. C. Beleites, U. Neugebauer, T. Bocklitz, C. Krafft, J. Popp. "Sample size planning for classification models". Analytica Chimica Acta. 2013. 760: 25-33. 10.1016/j.aca.2012.11.007.

149. M.J. Baker, H.J. Byrne, J. Chalmers, P. Gardner, R. Goodacre, A. Henderson, et al. "Clinical applications of infrared and Raman spectroscopy: state of play and future challenges". The Analyst. 2018. 143(8): 1735-1757. 10.1039/C7AN01871A.

150. D. Thomas. "Why the patents system needs a revamp". Raconteur. 2018. https://www.raconteur.net/legal/intellectual-property/patents-system/ [Feb 8 2021].

151. A. Hellström, S. Nilsson, M. Andersson, U. Håkanson. "Intellectual property for generating value for start-up companies in key enabling technologies". Biotechnology Research and Innovation. 2019. 3(1): 80-90. 10.1016/j.biori.2019.01.001.

152. R. Nikolowa. "Developing new ideas: Spin-outs, spinoffs, or internal divisions". Journal of Economic Behavior \& Organization. 2014. 98: 70-88. 10.1016/j.jebo.2013.12.001.

153. B. Clarysse, M. Wright, A. Lockett, E. Van de Velde, A. Vohora. "Spinning out new ventures: a typology of incubation strategies from European research institutions". Journal of Business Venturing. 2005. 20(2): 183-216. 10.1016/j.jbusvent.2003.12.004.

154. Medicines and Healthcare Products Regulatory Agency. Guidance of the In Vitro Diagonstic Medical Devices Directive 98/79/EC. UK Government, n.d.

155. A. Shiell. "Health economic evaluation". Journal of Epidemiology \& Community Health. 2002. 56(2): 85-88. 10.1136/jech.56.2.85.

156. D.P. Kernick. "Introduction to health economics for the medical practitioner". Postgraduate Medical Journal. 2003. 79(929): 147-150. 10.1136/pmj.79.929.147.

157. M. Drummond, M.J. Sculpher, K. Claxton, G.L. Stoddart, G.W. Torrance. Methods for the Economic Evaluation of Health Care Programmes: Oup Oxford, 2015.

158. S.R. Hill. "Cost-effectiveness analysis for clinicians". BMC Medicine. 2012. 10(1): 10. 10.1186/1741-7015-10-10.

159. S.J. Whitehead, S. Ali. "Health outcomes in economic evaluation: the QALY and utilities". British Medical Bulletin. 2010. 96(1): 5-21. 10.1093/bmb/ldq033.

160. B. Pham, H.A.T. Tu, D. Han, P. Pechlivanoglou, F. Miller, V. Rac, et al. "Early economic evaluation of emerging health technologies: protocol of a systematic review". Syst Rev. 2014. 3(1): 81. 10.1186/2046-4053-3-81.

161. E. Gray, H.J. Butler, R. Board, P.M. Brennan, A.J. Chalmers, T. Dawson, et al. "Health economic evaluation of a serum-based blood test for brain tumour diagnosis: exploration of two clinical scenarios". BMJ Open. 2018. 8(5): e017593. 10.1136/bmjopen-2017-017593.

162. J.R. Hands. Investigating the use of Attenuated Total Reflection Fourier-Transform Infrared (ATR-FTIR) Spectroscopy for the Rapid Diagnosis of Brain Tumours using Human Blood Serum. [Doctor of Philosophy]. University of Strathclyde, Glasgow, 2015.

163. B.R. Smith, K.M. Ashton, A. Brodbelt, T. Dawson, M.D. Jenkinson, N.T. Hunt, et al. "Combining random forest and 2D correlation analysis to identify serum spectral signatures for neuro-oncology". The Analyst. 2016. 141(12): 3668-3678. 10.1039/C5AN02452H. 
164. P.M. Brennan, H.J. Butler, L. Christie, M.G. Hegarty, M.D. Jenkinson, C. Keerie, et al. "Early detection of brain tumours using a novel spectroscopic liquid biopsy". Accepted Article Brain Communications. 2021.

165. E. Gray, J.M. Cameron, H.J. Butler, M.D. Jenkinson, M.G. Hegarty, D.S. Palmer, et al. "Early economic evaluation to guide the development of a spectroscopic liquid biopsy for the detection of brain cancer". Int J Technol Assess Health Care. 2021. 37: e41. $10.1017 / \mathrm{S} 0266462321000143$.

166. O. Stevens, I.E. Iping Petterson, J.C.C. Day, N. Stone. "Developing fibre optic Raman probes for applications in clinical spectroscopy". Chem. Soc. Rev. 2016. 45(7): 1919-1934. 10.1039/C5CS00850F.

167. M. Jermyn, K. Mok, J. Mercier, J. Desroches, J. Pichette, K. Saint-Arnaud, et al. "Intraoperative brain cancer detection with Raman spectroscopy in humans". Sci. Transl. Med. 2015. 7(274): 274ra19-274ra19. 10.1126/scitranslmed.aaa2384.

168. M. Jermyn, J. Mercier, K. Aubertin, J. Desroches, K. Urmey, J. Karamchandiani, et al. "Highly Accurate Detection of Cancer In Situ with Intraoperative, Label-Free, Multimodal Optical Spectroscopy". Cancer Res. 2017. 77(14): 3942-3950. 10.1158/0008-5472.CAN-17-0668.

169. L.J. Livermore, M. Isabelle, I. Mac Bell, C. Scott, J. Walsby-Tickle, J. Gannon, et al. "Rapid intraoperative molecular genetic classification of gliomas using Raman spectroscopy". NeuroOncology Advances. 2019. 1(1): 1-12. 10.1093/noajn1/vdz008.

170. C. Krafft, I.W. Schie, T. Meyer, M. Schmitt, J. Popp. "Developments in spontaneous and coherent Raman scattering microscopic imaging for biomedical applications". Chem. Soc. Rev. 2016. 45(7): 1819-1849. 10.1039/C5CS00564G.

171. I. Pence, A. Mahadevan-Jansen. "Clinical instrumentation and applications of Raman spectroscopy”. Chem. Soc. Rev. 2016. 45(7): 1958-1979. 10.1039/C5CS00581G.

172. A.F. García-Flores, L. Raniero, R.A. Canevari, K.J. Jalkanen, R.A. Bitar, H.S. Martinho, et al. "High-wavenumber FT-Raman spectroscopy for in vivo and ex vivo measurements of breast cancer". Theor Chem Acc. 2011. 130(4-6): 1231-1238. 10.1007/s00214-011-0925-9.

173. T.J.E. Hubbard, A.P. Dudgeon, D.J. Ferguson, A.C. Shore, N. Stone. "Utilization of Raman spectroscopy to identify breast cancer from the water content in surgical samples containing blue dye". Translational Biophotonics. 2021. 10.1002/tbio.202000023.

174. E.M. Barroso, R.W.H. Smits, T.C. Bakker Schut, I. ten Hove, J.A. Hardillo, E.B. Wolvius, et al. "Discrimination between Oral Cancer and Healthy Tissue Based on Water Content Determined by Raman Spectroscopy". Anal. Chem. 2015. 87(4): 2419-2426. 10.1021/ac504362y.

175. Y. Aaboubout, I. ten Hove, R.W.H. Smits, J.A. Hardillo, G.J. Puppels, S. Koljenovic. "Specimen-driven intraoperative assessment of resection margins should be standard of care for oral cancer patients". Oral Dis. 2021. 27(1): 111-116. 10.1111/odi.13619.

176. L. Avram, A. Stefancu, D. Crisan, N. Leopold, V. Donca, E. Buzdugan, et al. "Recent advances in surface-enhanced Raman spectroscopy based liquid biopsy for colorectal cancer (Review)". Exp Ther Med. 2020. 20(6): 1-1. 10.3892/etm.2020.9342.

177. F. Nicolson, B. Andreiuk, C. Andreou, H.-T. Hsu, S. Rudder, M.F. Kircher. "Non-invasive In Vivo Imaging of Cancer Using Surface-Enhanced Spatially Offset Raman Spectroscopy (SESORS)". Theranostics. 2019. 9(20): 5899-5913. 10.7150/thno.36321.

178. Y. Du, G. Lv, H. Li, D. Tong, X. Lv, Z. Zhang, et al. "Quantitative analysis of hepatitis B virus DNA based on raman spectroscopy combined with multivariate statistical methods". Laser Phys. Lett. 2020. 17(2): 025001. 10.1088/1612-202X/ab6855.

179. CRIMSON. "Coherent Raman Imaging for the Molecular Study of the Origin of Diseases". n.d. https://www.crimson-project.eu/ [Feb 3 2021].

180. CRIMSON. "Press Release: European consortium to build next-generation microscope promising to revolutionize the study of the cellular origin of diseases." 2020. https://www.crimson-project.eu/news/ [Feb 3 2021].

181. Raman Nanotheranostics. "The Raman Nanotheranostics (RaNT)". n.d. https://rantmedicine.com/about-rant/ [Feb 8 2021].

182. RiverD International B.V. "RiverD”. n.d. https://www.riverd.com/ [Feb 3 2021]. 
183. P.J. Caspers, C. Nico, T.C. Bakker Schut, J. Sterke, P.D.A. Pudney, P.R. Curto, et al. "Method to quantify the in vivo skin penetration of topically applied materials based on confocal RAMAN spectroscopy". Translational Biophotonics. 2019. 1(1-2). 10.1002/tbio.201900004.

184. S. Guo, C. Beleites, U. Neugebauer, S. Abalde-Cela, N.K. Afseth, F. Alsamad, et al. "Comparability of Raman Spectroscopic Configurations: A Large Scale Cross-Laboratory Study". Anal. Chem. 2020. 92(24): 15745-15756. 10.1021/acs.analchem.0c02696.

185. S. Fornasaro, F. Alsamad, M. Baia. "Surface Enhanced Raman Spectroscopy for Quantitative Analysis: Results of a Large-Scale European Multi-Instrument Interlaboratory Study". Anal. Chem. 2020. 12.

186. H.J. Butler, P.M. Brennan, J.M. Cameron, D. Finlayson, M.G. Hegarty, M.D. Jenkinson, et al. "Development of high-throughput ATR-FTIR technology for rapid triage of brain cancer". Nat Commun. 2019. 10(1): 4501. 10.1038/s41467-019-12527-5.

187. S. Roy, D. Perez-Guaita, D.W. Andrew, J.S. Richards, D. McNaughton, P. Heraud, et al. "Simultaneous ATR-FTIR Based Determination of Malaria Parasitemia, Glucose and Urea in Whole Blood Dried onto a Glass Slide". Analytical Chemistry. 2017. 89(10): 5238-5245. 10.1021/acs.analchem.6b04578.

188. P. Heraud, P. Chatchawal, M. Wongwattanakul, P. Tippayawat, C. Doerig, P. Jearanaikoon, et al. "Infrared spectroscopy coupled to cloud-based data management as a tool to diagnose malaria: a pilot study in a malaria-endemic country". Malar J. 2019. 18(1): 348. 10.1186/s12936-019-2945-1.

189. M. Martin, D. Perez-Guaita, D.W. Andrew, J.S. Richards, B.R. Wood, P. Heraud. "The effect of common anticoagulants in detection and quantification of malaria parasitemia in human red blood cells by ATR-FTIR spectroscopy". Analyst. 2017. 142(8): 1192-1199. 10.1039/C6AN02075E.

190. A. Khoshmanesh, M.W.A. Dixon, S. Kenny, L. Tilley, D. McNaughton, B.R. Wood. "Detection and Quantification of Early-Stage Malaria Parasites in Laboratory Infected Erythrocytes by Attenuated Total Reflectance Infrared Spectroscopy and Multivariate Analysis". Anal. Chem. 2014. 86(9): 4379-4386. 10.1021/ac500199x.

191. D. Perez-Guaita, K.M. Marzec, A. Hudson, C. Evans, T. Chernenko, C. Matthäus, et al. "Parasites under the Spotlight: Applications of Vibrational Spectroscopy to Malaria Research". Chemical Reviews. 2018. 118(11): 5330-5358. 10.1021/acs.chemrev.7b00661.

192. T. Nitrosetein, M. Wongwattanakul, C. Chonanant, C. Leelayuwat, N. Charoensri, P. Jearanaikoon, et al. "Attenuated Total Reflection Fourier Transform Infrared Spectroscopy combined with chemometric modelling for the classification of clinically relevant Enterococci”. J Appl Microbiol. 2020. jam.14820. 10.1111/jam.14820.

193. S. Pebotuwa, K. Kochan, A. Peleg, B.R. Wood, P. Heraud. "Influence of the Sample Preparation Method in Discriminating Candida spp. Using ATR-FTIR Spectroscopy". Molecules. 2020. 25(7): 1551. 10.3390/molecules25071551.

194. Biotech Resources. "Rapid sepsis diagnostic receives funding to boost health innovations in Australia". 2018. https://www.btrdiagnostics.com/biomedtech-horizons-grant [Feb 3 2021].

195. K. Kochan, C. Nethercott, J. Taghavimoghaddam, Z. Richardson, E. Lai, S.A. Crawford, et al. "Rapid Approach for Detection of Antibiotic Resistance in Bacteria Using Vibrational Spectroscopy". Anal. Chem. 2020. 92(12): 8235-8243. 10.1021/acs.analchem.0c00474.

196. Glyconics. "A major advance in point-of-care testing". n.d. http://www.glyconics.com/\#technology [Feb 32021$]$.

197. R. Coopman, T. Van de Vyver, A.S. Kishabongo, P. Katchunga, E.H. Van Aken, J. Cikomola, et al. "Glycation in human fingernail clippings using ATR-FTIR spectrometry, a new marker for the diagnosis and monitoring of diabetes mellitus". Clinical Biochemistry. 2017. 50(1-2): 62-67. 10.1016/j.clinbiochem.2016.09.001.

198. T. Monteyne, R. Coopman, A.S. Kishabongo, J. Himpe, B. Lapauw, S. Shadid, et al. "Analysis of protein glycation in human fingernail clippings with near-infrared (NIR) spectroscopy as an alternative technique for the diagnosis of diabetes mellitus". Clinical Chemistry and Laboratory Medicine (CCLM). 2018. 56(9): 1551-1558. 10.1515/cclm-2018-0239. 
199. A.S. Kishabongo, P. Katchunga, E.H. Van Aken, M.M. Speeckaert, S. Lagniau, D. Husein, et al. "Glycated nail proteins: a new approach for detecting diabetes in developing countries". Trop Med Int Health. 2014. 19(1): 58-64. 10.1111/tmi.12218.

200. D. Mayerich, M.J. Walsh, A. Kadjacsy-Balla, P.S. Ray, S.M. Hewitt, R. Bhargava. "Stain-less staining for computed histopathology”. Technology. 2015. 03(01): 27-31. $10.1142 / \mathrm{S} 2339547815200010$.

201. M.J. Baker, J. Trevisan, P. Bassan, R. Bhargava, H.J. Butler, K.M. Dorling, et al. "Using Fourier transform IR spectroscopy to analyze biological materials". Nature Protocols. 2014. 9(8): 1771-1791. 10.1038/nprot.2014.110.

202. M. Diem, A. Ergin, X. Mu. "Spectral histopathology of the lung: A review of two large studies". J. Biophotonics. 2019. 12(9). 10.1002/jbio.201900061.

203. R. Bangaoil, A. Santillan, L.M. Angeles, L. Abanilla, A. Lim, Ma.C. Ramos, et al. "ATR-FTIR spectroscopy as adjunct method to the microscopic examination of hematoxylin and eosinstained tissues in diagnosing lung cancer". Y. Garini, editor. PLoS ONE. 2020. 15(5): e0233626. 10.1371/journal.pone.0233626.

204. J.A. de A.C. Piva, J.L.R. Silva, L.J. Raniero, C.S.P. Lima, E.A.L. Arisawa, C. de Oliveira, et al. "Biochemical imaging of normal, adenoma, and colorectal adenocarcinoma tissues by Fourier transform infrared spectroscopy (FTIR) and morphological correlation by histopathological analysis: preliminary results". Res. Biomed. Eng. 2015. 31(1): 10-18. 10.1590/2446-4740.0321.

205. S. Brézillon, V. Untereiner, H.T. Mohamed, E. Ahallal, I. Proult, P. Nizet, et al. "Label-Free Infrared Spectral Histology of Skin Tissue Part II: Impact of a Lumican-Derived Peptide on Melanoma Growth". Front. Cell Dev. Biol. 2020. 8: 377. 10.3389/fcell.2020.00377.

206. M.H.M. Ali, F. Rakib, K. Al-Saad, R. Al-Saady, E. Goormaghtigh. "An Innovative Platform Merging Elemental Analysis and Ftir Imaging for Breast Tissue Analysis". Sci Rep. 2019. 9(1): 9854. 10.1038/s41598-019-46056-4.

207. M. Pilling, P. Gardner. "Fundamental developments in infrared spectroscopic imaging for biomedical applications". Chem. Soc. Rev. 2016. 45(7): 1935-1957. 10.1039/C5CS00846H.

208. G. Clemens, B. Bird, M. Weida, J. Rowlette, M.J. Baker. "Quantum cascade laser-based midinfrared spectrochemical imaging of tissue and biofluids". Spectroscopy Europe. 2014. 26(4): $14-19$.

209. A. Schwaighofer, M. Brandstetter, B. Lendl. "Quantum cascade lasers (QCLs) in biomedical spectroscopy”. Chem. Soc. Rev. 2017. 46(19): 5903-5924. 10.1039/C7CS00403F.

210. P. Bassan, M.J. Weida, J. Rowlette, P. Gardner. "Large scale infrared imaging of tissue micro arrays (TMAs) using a tunable Quantum Cascade Laser (QCL) based microscope". Analyst. 2014. 139(16): 3856-3859. 10.1039/C4AN00638K.

211. M. Hermes, R.B. Morrish, L. Huot, L. Meng, S. Junaid, J. Tomko, et al. "Mid-IR hyperspectral imaging for label-free histopathology and cytology". J. Opt. 2018. 20(2): 023002. 10.1088/2040-8986/aaa36b.

212. M.J. Pilling, A. Henderson, P. Gardner. "Quantum Cascade Laser Spectral Histopathology: Breast Cancer Diagnostics Using High Throughput Chemical Imaging”. Anal. Chem. 2017. 89(14): 7348-7355. 10.1021/acs.analchem.7b00426.

213. C. Kuepper, A. Kallenbach-Thieltges, H. Juette, A. Tannapfel, F. Großerueschkamp, K. Gerwert. "Quantum Cascade Laser-Based Infrared Microscopy for Label-Free and Automated Cancer Classification in Tissue Sections". Sci Rep. 2018. 8(1): 7717. 10.1038/s41598-01826098-w.

214. M. Schnell, S. Mittal, K. Falahkheirkhah, A. Mittal, K. Yeh, S. Kenkel, et al. "All-digital histopathology by infrared-optical hybrid microscopy". Proc Natl Acad Sci USA. 2020. 117(7): 3388-3396. 10.1073/pnas.1912400117.

215. J.F. Cohen, D.A. Korevaar, D.G. Altman, D.E. Bruns, C.A. Gatsonis, L. Hooft, et al. "STARD 2015 guidelines for reporting diagnostic accuracy studies: explanation and elaboration". BMJ Open. 2016. 6(11): e012799. 10.1136/bmjopen-2016-012799. 


\section{Figure Legend}

Figure 1 - Evolution of publications from a web of knowledge search for "FTIR" and ("clinical" or "biology" or "medicine") or "Raman" and ("clinical" or "biology" or "medicine") from 1978-2020.

Figure 2 - Conceptual framework for visualising translation of biomedical research. Adapted from ref. ${ }^{15}$ with permission from Springer Nature.

Figure 3 - Example of assigning Raman spectra to tissue structures and ductal carcinoma assigned by twostep k-means clustering: (a) and (b) samples with invasive ductal carcinoma, (c) normal breast tissue. Typical centroid Raman spectra are shown for the clusters indicated by arrows: focus of invasive ductal carcinoma (red arrows), tumour-surrounding inflammatory stroma (green arrows), lobules and ducts (blue arrows), stroma (black arrows) and fat (orange arrows). Reproduced from ref. ${ }^{54}$ under a Creative Commons Attribution 3.0 licence.

Figure 4 - The handheld contact fibre optic probe for Raman spectroscopy utilised by Jermyn et al.: (a) Experimental setup diagram with the 785-nm near-IR laser and the high-resolution charge-coupled device spectroscopic detector used with the Raman fibre optic probe; BP, band-pass; LP, long-pass. (b) The probe was used to interrogate brain tissue during surgery. A schematic diagram illustrates the excitation of different molecular species, such as cholesterol and DNA, to produce the Raman spectra of cancer versus normal brain tissue. A simple molecular vibrational mode is conceptually depicted (individual atoms in blue and green) interacting with the laser light (in red) to produce Raman scattering (in purple). Reproduced from ref. ${ }^{167}$ with permission from AAAS.

Figure 5 - Infrared-optical hybrid (IR-OH) microscopy imaging of breast tissue and spectral cell type recognition. (A) Haematoxylin and eosin image of a breast tissue microarray section; (B) IR-OH absorption of an adjacent, unstained tissue section at $1550 \mathrm{~cm}^{-1}$; (C) A four-class model (blood, epithelium, stroma, and other) allows rapid tissue component visualization based on five IR bands; (D) Epithelial classification (five-class model) permits both histologic cellular identification and recognition of cancer based on seven IR bands; (E) the ROC curves related to the classes in D; (F) the AUC of the ROC curve increases with number of IR frequencies used for classification; ( $\mathrm{G}$ and $\mathrm{H}$ ) Representative class spectra obtained with IR-OH and FT-IR imaging, respectively. Adapted from ref. ${ }^{214}$ under the PNAS License to Publish. 
Figure 1:

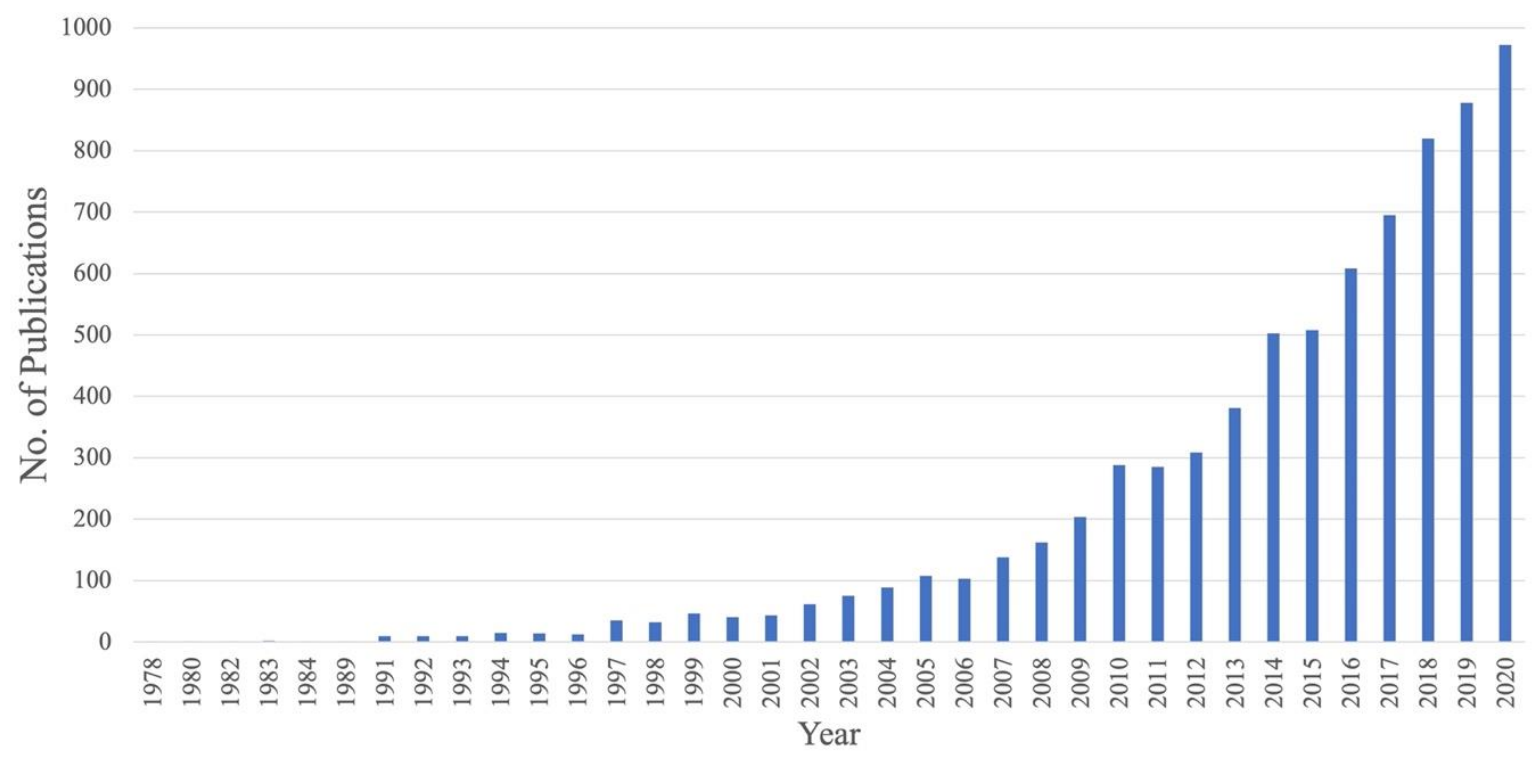

Figure 2:

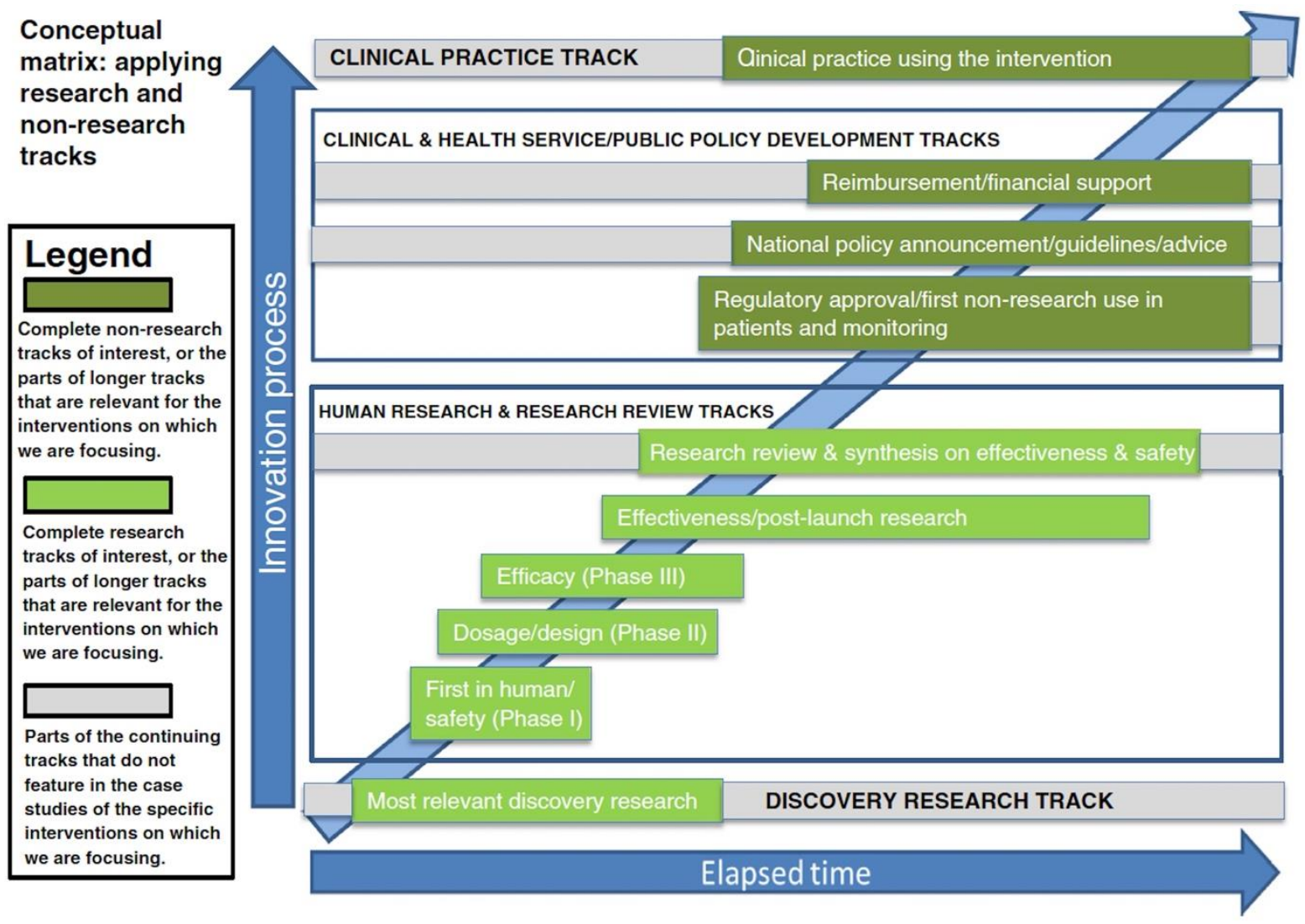


Figure 3:

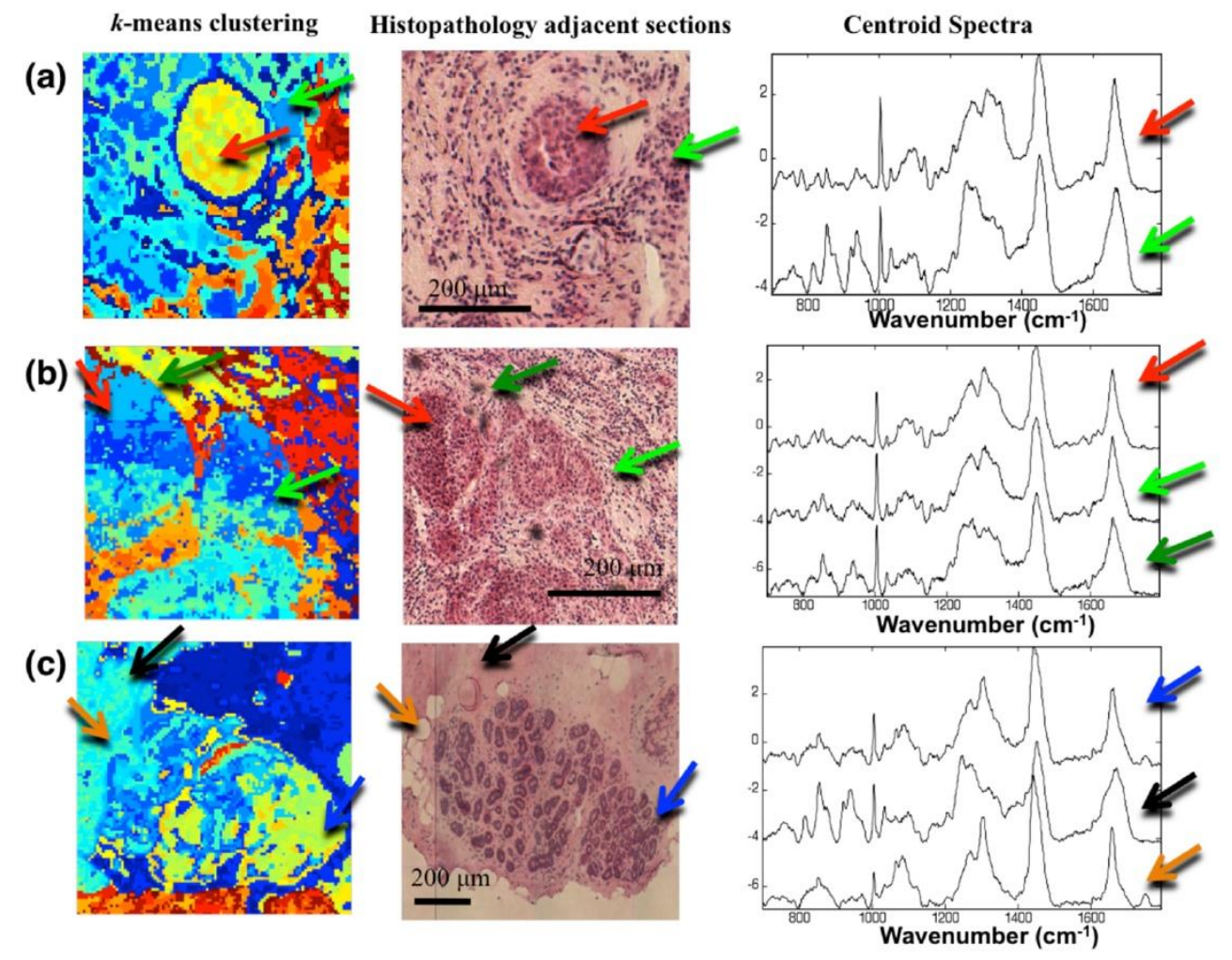

Figure 4:

(a)

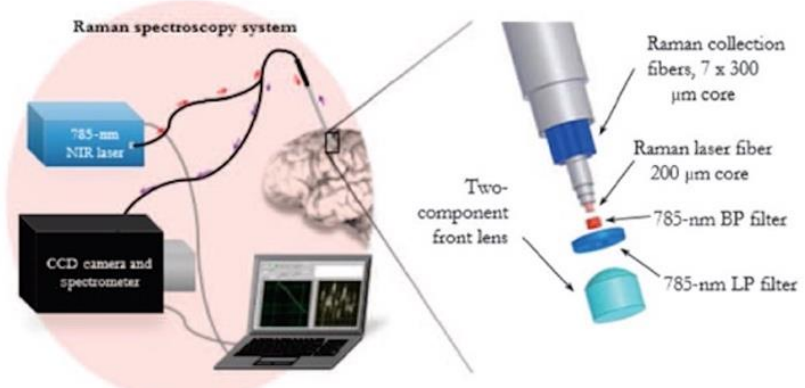

(b)

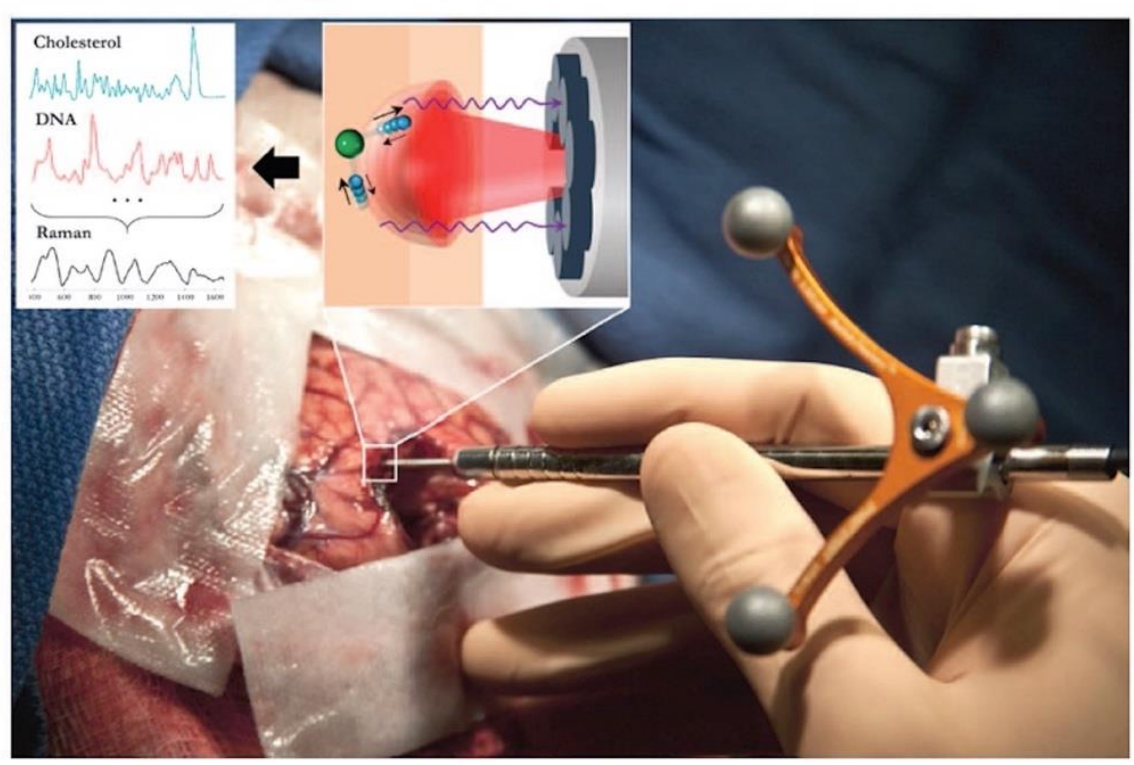


Figure 5:

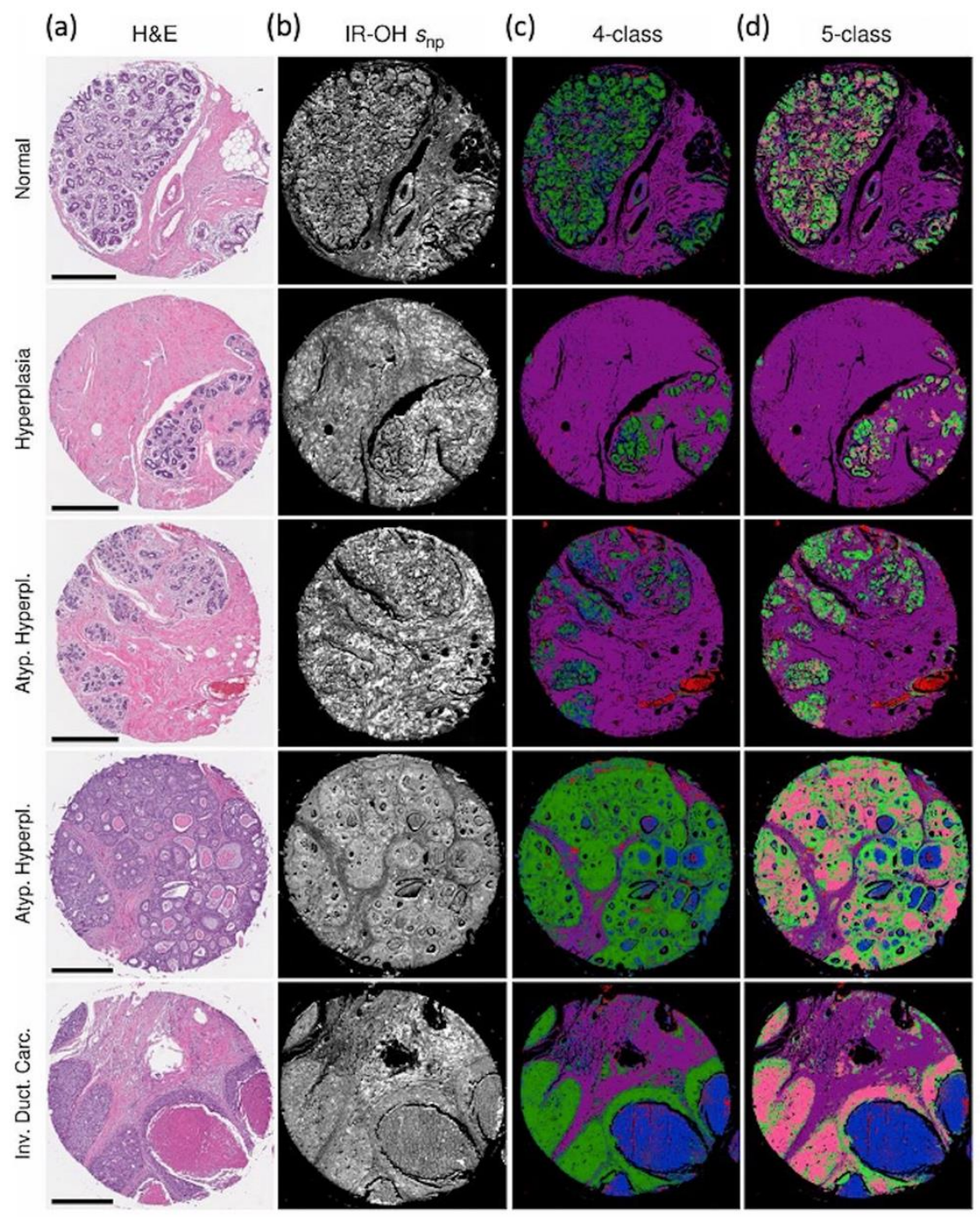

(e)
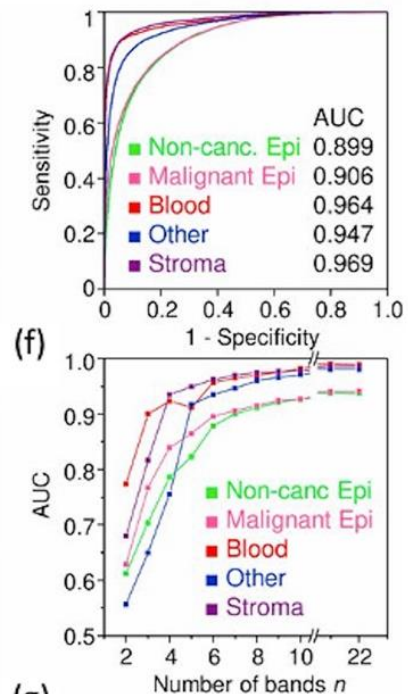

(g)
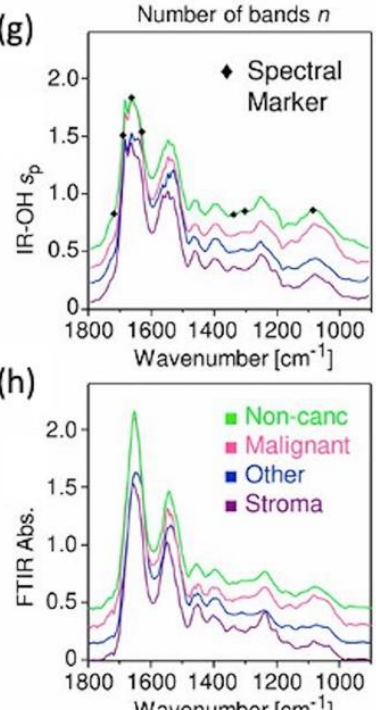